\title{
Electron-muon heat conduction in neutron star cores via the exchange of transverse plasmons
}

\author{
P. S. Shternin and D. G. Yakovlev \\ Ioffe Physico-Technical Institute, Politekhnicheskaya 26, 194021 Saint-Petersburg, Russia
}

(Dated: October 24, 2018)

\begin{abstract}
We calculate the thermal conductivity of electrons and muons $\kappa_{e \mu}$ produced owing to electromagnetic interactions of charged particles in neutron star cores and show that these interactions are dominated by the exchange of transverse plasmons (via the Landau damping of these plasmons in nonsuperconducting matter and via a specific plasma screening in the presence of proton superconductivity). For normal protons, the Landau damping strongly reduces $\kappa_{e \mu}$ and makes it temperature independent. Proton superconductivity suppresses the reduction and restores the Fermi-liquid behavior $\kappa_{e \mu} \propto T^{-1}$. Comparing with the thermal conductivity of neutrons $\kappa_{n}$, we obtain $\kappa_{e \mu} \gtrsim \kappa_{n}$ for $T \gtrsim 2 \times 10^{9} \mathrm{~K}$ in normal matter and for any $T$ in superconducting matter with proton critical temperatures $T_{c p} \gtrsim 3 \times 10^{9} \mathrm{~K}$. The results are described by simple analytic formulae.

PACS numbers: 52.25.Fi, 95.30.Tg, 97.20.Rp, 97.60.Jd
\end{abstract}

\section{INTRODUCTION}

Neutron stars are compact stars of mass $\sim 1.4 M_{\odot}$ and radius $\sim 10-15 \mathrm{~km}$, where $M_{\odot}$ is the solar mass. A neutron star is thought to consist of a thin crust $(\sim 1 \%$ by mass) and a bulky core [1, 2, 3, 4]. The crust is composed of a nonuniform matter containing atomic nuclei. The core extends from the layer of the density $\rho \approx 0.5 \rho_{0}$ to the stellar center $\left[\rho \sim(10-20) \rho_{0}\right]$, where $\rho_{0} \approx 2.8 \times 10^{14} \mathrm{~g} \mathrm{~cm}^{-3}$ is the mass density of saturated symmetric nuclear matter. It is widely thought that the core is built of neutron-rich, strongly degenerate, uniform nuclear matter of supernuclear density. This makes neutron stars unique natural laboratories of such matter. The composition and equation of state (EOS) of this matter are largely unknown but will hopefully be strongly constrained soon by comparing theoretical models of neutron star structure and evolution with observations.

We will consider the thermal conductivity of neutron star cores. The thermal conductivity of neutron stars is needed for modeling the thermal structure and evolution of these stars and related phenomena [5, 6]. In particular, it is important for cooling of isolated neutron stars and for thermal structure of transiently accreting neutron stars (in soft X-ray transients). Accreting stars can be heated by pycnonuclear reactions when accreted matter sinks into the deep neutron star crust under the weight of newly accreted material [7, 8, 9]. Thermal conduction spreads this heat over the star - to the surface (resulting in surface photon emission) and to the core (producing neutrino emission from the core). In this way the observable surface thermal emission depends on the neutrino luminosity of the star, and, hence, on its internal structure. The thermal conductivity of neutron star cores is especially important for studying cooling of young neutron stars (of age $t \lesssim 100$ years), where the internal thermal relaxation is not yet achieved (e.g., Refs. [10, 11]). The thermal conductivity can also regulate thermal relaxation of a neutron star in response to pulsar glitches (see, e.g., [12], and references therein).
We will focus on models of neutron star cores composed of neutrons $(n)$, with an admixture of protons $(p)$, electrons $(e)$, and muons $(\mu)$. Our results are easily generalized for matter containing hyperons. In all these cases, the thermal conductivity $\kappa$ can be subdivided into two relatively independent parts [13, 14],

$$
\kappa \approx \kappa_{b}+\kappa_{e \mu},
$$

where $\kappa_{b}$ is the conductivity of neutrons (and possibly other baryons) mediated by strong-interaction collisions of heat-conducting particles (see, e.g., [15], and references therein), while $\kappa_{e \mu}$ is the conductivity of electrons and muons mediated by electromagnetic interactions. The conductivity $\kappa_{e \mu}$ has been calculated in several papers, particularly, in Refs. [13, 16, 17].

However, all previous calculations of $\kappa_{e \mu}$ in neutron star cores have neglected an important effect of the Landau damping of electromagnetic interactions owing to the exchange of transverse plasmons. In the context of transport properties of dense matter this effect was studied by Heiselberg and Pethick [18] for a degenerate quark plasma. Recently we have included the effect of the Landau damping into the thermal conductivity of strongly degenerate electrons determined by electron-electron collisions with the electron plasma screening [19]. Similar effects have also been studied by Jaikumar et al. 20] for neutrino bremsstrahlung radiation via electron-electron collisions in neutron star crusts and cores. Here we reconsider $\kappa_{e \mu}$ in a neutron star core including the effects of the Landau damping and of the specific plasma screening induced by proton superconductivity.

\section{ELECTRON-MUON THERMAL CONDUCTIVITY IN NORMAL MATTER}

The electrons and muons in a neutron star core constitute strongly degenerate almost ideal Fermi gases [1]. The electrons are ultrarelativistic. The muons can be 
absent in the outermost part of the core; they are nonrelativistic just after their creation threshold and become relativistic at higher densities. The neutrons and protons constitute strongly interacting Fermi liquids. The neutrons are mainly nonrelativistic (although they become mildly relativistic near the center of a massive neutron star); the less abundant protons are typically nonrelativistic. The neutrons and protons can be in superfluid states; superfluidity of protons means their superconductivity. In this section we consider nonsuperfluid (normal) matter. We analyze the effects of proton superconductivity in Sec. III. We closely follow the derivation of $\kappa_{e \mu}$ by Gnedin and Yakovlev [17] (but introduce the Landau damping); thus we omit technical details.

The thermal conductivity $\kappa_{e \mu}$ is calculated from a coupled system of linearized Boltzmann equations for the electron and muon distribution functions $\widetilde{f}_{e}$ and $\widetilde{f}_{\mu}$, which we denote collectively by $\widetilde{f}_{c}$, with $c=e$ or $\mu$. These distributions slightly deviate from the equilibrium Fermi-Dirac distributions $f_{c}$ owing to the presence of a weak temperature gradient $\nabla T$,

$$
\begin{aligned}
& \tilde{f}_{c}=f_{c}-\Phi_{c} \frac{\partial f_{c}}{\partial \varepsilon_{c}} \\
& f_{c}=\left\{\exp \left(\frac{\varepsilon_{c}-\mu_{c}}{k_{B} T}\right)+1\right\}^{-1},
\end{aligned}
$$

where $\varepsilon_{c}$ is the particle energy, $\mu_{c}$ is its chemical potential (the rest-mass term included), $T$ is the temperature, $k_{B}$ the Boltzmann constant, and $\Phi_{c}$ measures a deviation from equilibrium. For calculating $\kappa_{e \mu}$, the electrons and muons are treated as the only heat carriers which experience electromagnetic interactions between themselves and with any charged baryons in dense matter (with protons, in our case). The charged baryons are assumed to obey equilibrium Fermi-Dirac distributions (see, e.g., Ref. 17]). Nonequilibrium parts of the electron and muon distributions are found using the standard variational approach with the simplest trial function,

$$
\Phi_{c}=-\tau_{c}\left(\varepsilon_{c}-\mu_{c}\right) \boldsymbol{v}_{c} \cdot \nabla T / T,
$$

where $\boldsymbol{v}_{c}$ is the velocity of particles $c$ and $\tau_{c}$ is their effective relaxation time.

The resulting electron and muon thermal conductivity can be written [17] in a familiar form

$$
\begin{aligned}
& \kappa_{e \mu}=\kappa_{e}+\kappa_{\mu}, \\
& \kappa_{e}=\frac{\pi^{2} k_{B}^{2} T n_{e} \tau_{e}}{3 m_{e}^{*}}, \quad \kappa_{\mu}=\frac{\pi^{2} k_{B}^{2} T n_{\mu} \tau_{\mu}}{3 m_{\mu}^{*}},
\end{aligned}
$$

where $\kappa_{e}$ and $\kappa_{\mu}$ are the partial thermal conductivities of electrons and muons, respectively; $n_{e}$ and $n_{\mu}$ are number densities of these particles; $m_{e}^{*}=\mu_{e} / c^{2}$ and $m_{\mu}^{*}=\mu_{\mu} / c^{2}$ are their effective masses. In neutron star cores at beta equilibrium one has $\mu_{e}=\mu_{\mu}$ and $m_{e}^{*}=m_{\mu}^{*}$ [1]. Note that the thermal conductivities $\kappa_{e}$ and $\kappa_{\mu}$ presented by Gnedin and Yakovlev [17] [their Eqs. (61) and (65)] contain also an additional factor $C \approx 1.2$ which brings the employed simplest variational solution closer to the exact solution. We do not introduce a similar correction here. Its calculation is more complicated than in Ref. [17], because now we include the Landau damping of transverse plasmons. The Landau damping introduces non-Fermiliquid behavior into the thermal conductivity (Sec. IIB), while the standard technique for calculating the corrections is developed for traditional Fermi liquids. In Sec. IIC we argue that the Landau damping of transverse plasmons reduces the difference between the exact and simplest variational solutions. Thus, neglecting the correction factor $C$ in the expression for $\kappa_{e \mu}$ can introduce uncertainties $\lesssim 20 \%$ which are quite acceptable for the thermal conduction problem in neutron star cores.

The effective electron and muon relaxation times can be written as [17]

$$
\tau_{e}=\frac{\nu_{\mu}-\nu_{e \mu}^{\prime}}{\nu_{e} \nu_{\mu}-\nu_{e \mu}^{\prime} \nu_{\mu e}^{\prime}}, \quad \tau_{\mu}=\frac{\nu_{e}-\nu_{\mu e}^{\prime}}{\nu_{e} \nu_{\mu}-\nu_{e \mu}^{\prime} \nu_{\mu e}^{\prime}},
$$

where

$$
\begin{aligned}
\nu_{e} & =\sum_{i} \nu_{e i}=\nu_{e e}+\nu_{e \mu}+\nu_{e p}, \\
\nu_{\mu} & =\sum_{i} \nu_{\mu i}=\nu_{\mu \mu}+\nu_{\mu e}+\nu_{\mu p}
\end{aligned}
$$

are the total effective collision frequencies of electrons and muons with all charged particles $i$ (which are electrons, muons and protons, in our case); $\nu_{e i}$ and $\nu_{\mu i}$ are familiar partial effective collision frequencies (derived in a standard manner from respective linearized collision integrals), while $\nu_{e \mu}^{\prime}$ and $\nu_{\mu e}^{\prime}$ are two additional effective collision frequencies 17. which couple heat transport of the electrons and muons. All these collision frequencies can be expressed as multi-dimensional integrals over momenta of colliding particles. Since all particles are strongly degenerate, their momenta can be placed at appropriate Fermi surfaces whenever possible. In this way, for collisions of nonidentical particles 1 and 2 we obtain

$$
\begin{aligned}
& \nu_{12}=\frac{3}{T^{3} v_{F 1} p_{F 1}^{2}}\left(S_{11}-S_{11^{\prime}}\right), \\
& \nu_{12}^{\prime}=\frac{3}{T^{3} v_{F 1} p_{F 1}^{2}}\left(S_{12}-S_{12^{\prime}}\right),
\end{aligned}
$$

while for collisions between identical heat carriers we have $\nu_{c c}=\nu_{12}+\nu_{12}^{\prime}$. Here, $v_{F 1}$ and $p_{F 1}$ are, respectively, the Fermi velocity and the Fermi momentum of particles 1;

$$
\begin{aligned}
S_{\alpha \beta}= & (2 \pi)^{-12} \int \mathrm{d}^{3} p_{1} \mathrm{~d}^{3} p_{1}^{\prime} \mathrm{d}^{3} p_{2} \mathrm{~d}^{3} p_{2}^{\prime} \\
& \times W\left(1,2 \mid 1^{\prime}, 2^{\prime}\right) f_{1} f_{2}\left(1-f_{1}^{\prime}\right)\left(1-f_{2}^{\prime}\right) \\
& \times\left(\boldsymbol{v}_{\alpha} \cdot \boldsymbol{v}_{\beta}\right)\left(\varepsilon_{\alpha}-\mu_{\alpha}\right)\left(\varepsilon_{\beta}-\mu_{\beta}\right)
\end{aligned}
$$

is the normalized collision frequency;

$$
\begin{aligned}
W\left(1,2 \mid 1^{\prime}, 2^{\prime}\right)= & 4(2 \pi)^{4} \delta\left(\varepsilon_{1}+\varepsilon_{2}-\varepsilon_{1}^{\prime}-\varepsilon_{2}^{\prime}\right) \\
& \times \delta\left(\boldsymbol{p}_{1}+\boldsymbol{p}_{2}-\boldsymbol{p}_{1}^{\prime}-\boldsymbol{p}_{2}^{\prime}\right)\left|M_{12}\right|^{2}
\end{aligned}
$$


is the differential transition probability for a scattering process $\boldsymbol{p}_{1} \boldsymbol{p}_{2} \rightarrow \boldsymbol{p}_{1}^{\prime} \boldsymbol{p}_{2}^{\prime} ;\left|M_{12}\right|^{2}$ is the squared matrix element summed over particle spin states (it includes also the symmetry factor to avoid double counting of the same collisions events); primes refer to particles after the collision. In Eqs. (7) - (9) we use the system of units in which $c=k_{\mathrm{B}}=\hbar=1$. The same system will be used below unless the contrary is indicated.

In the absence of muons we have

$$
\kappa_{e \mu}=\kappa_{e}, \quad \tau_{e}^{-1}=\nu_{e}=\nu_{e e}+\nu_{e p}
$$

\section{A. Dynamical screening of electromagnetic interaction}

The physics of the dynamical plasma screening is thoroughly analyzed by Heiselberg and Pethick [18]. These authors consider quark-quark collisions in a quark plasma through one-gluon exchange in the weak-coupling limit. Such collisions are similar to electromagnetic scattering of charged particles in an ordinary plasma. Electromagnetic interactions of muons and electrons in neutron star cores are usually accompanied by small momentum and energy transfers which greatly simplifies the theory. The squared matrix element for small energy transfers in a collision $\boldsymbol{p}_{1} \boldsymbol{p}_{2} \rightarrow \boldsymbol{p}_{1}^{\prime} \boldsymbol{p}_{2}^{\prime}$ of nonidentical particles 1 and 2 is

$$
\left|M_{12}\right|^{2} \propto\left|\frac{J_{1^{\prime} 1}^{(0)} J_{2^{\prime} 2}^{(0)}}{q^{2}+\Pi_{l}}-\frac{\boldsymbol{J}_{t 1^{\prime} 1} \cdot \boldsymbol{J}_{t 2^{\prime} 2}}{q^{2}-\omega^{2}+\Pi_{t}}\right|^{2}
$$

where $\hbar \boldsymbol{q}=\boldsymbol{p}_{1}^{\prime}-\boldsymbol{p}_{1}$ is a momentum transfer, $\hbar \omega=$ $\varepsilon_{1}^{\prime}-\varepsilon_{1}$ is an energy transfer (in standard physical units), $J_{i}^{(\nu)}=\left(J_{i^{\prime} i}^{(0)}, \boldsymbol{J}_{i^{\prime} i}\right)=\left(\bar{u}_{i}^{\prime} \gamma^{\nu} u_{i}\right)$ is the transition 4-current $(\nu=0, \ldots, 3), \gamma^{\nu}$ is a Dirac matrix, $u_{i}$ is a normalized bispinor $\left(\bar{u}_{i} u_{i}=2 m_{i}\right), \bar{u}_{i}$ is a Dirac conjugate (see, e.g., Berestetskil, Lifshitz and Pitaevskii [21]); $\boldsymbol{J}_{t i^{\prime} i}$ is the component of $\boldsymbol{J}_{i^{\prime} i}$ transverse to $\boldsymbol{q}$. The longitudinal component of $\boldsymbol{J}_{i^{\prime} i}$ (parallel to $\boldsymbol{q}$ ) is related to the time-like (charge density) component $J_{i^{\prime} i}^{(0)}$ via charge conservation. It is excluded from Eq. (11) with the aid of the continuity equation as explained by Heiselberg and Pethick [18]. For collisions of identical particles ( $e e$ and $\mu \mu$ in our case), the matrix element contains two parts, $M_{12}=M_{12}^{(1)}+M_{12}^{(2)}$, which correspond to two channels, $\left(1 \rightarrow 1^{\prime} ; 2 \rightarrow 2^{\prime}\right)$ and $\left(1 \rightarrow 2^{\prime} ; 1^{\prime} \rightarrow 2\right)$. However, in the small-momentumtransfer approximation, the interference term is small, both channels give equal contributions, and the relationship (11) is not violated.

Equation (11) contains the polarization functions $\Pi_{l}$ and $\Pi_{t}$ which depend on $\omega$ and $q$ and describe plasma screening of interparticle interaction by longitudinal and transverse plasma perturbations (plasmons), respectively. In the random phase approximation (RPA), these functions are the sums of terms for all charged particles $i$ (electrons, muons and protons). In the classical limit $\left(q \ll p_{F i}, \omega \ll v_{F i} p_{F i}\right)$ one has (e.g., Alexandrov, Bogdankevich and Rukhadze [22])

$$
\begin{aligned}
& \Pi_{l}=\sum_{i} \frac{4 \alpha p_{F i}^{2}}{\pi v_{F i}} \chi_{l}\left(x_{i}\right), \\
& \Pi_{t}=\sum_{i} \frac{4 \alpha p_{F i}^{2} v_{F i}}{\pi} \chi_{t}\left(x_{i}\right),
\end{aligned}
$$

where $x_{i}=\omega /\left(q v_{F i}\right), \alpha=e^{2} / \hbar c \approx 1 / 137$ is the fine structure constant, and

$$
\begin{aligned}
& \chi_{l}(x)=1-\frac{x}{2} \ln \left(\frac{x+1}{x-1}\right) \\
& \chi_{t}(x)=\frac{x^{2}}{2}+\frac{x\left(1-x^{2}\right)}{4} \ln \left(\frac{x+1}{x-1}\right) .
\end{aligned}
$$

For typical conditions of very strong degeneracy in neutron star cores, it is sufficient to use the expressions for $\Pi_{l}$ and $\Pi_{t}$ in the limit of $q \ll p_{F i}$ and $x_{i} \ll 1$, in which $\chi_{l}(x) \approx 1$ and $\chi_{t}(x) \approx i \pi x / 4$. In this limit,

$$
\begin{aligned}
& \Pi_{l}=\sum_{i} \frac{3 \omega_{i}^{2}}{v_{F i}^{2}}=q_{l}^{2}, \\
& \Pi_{t}=i \frac{\pi}{4} \frac{\omega}{q} \sum_{i} \frac{3 \omega_{i}^{2}}{v_{F i}}=i \frac{\pi}{4} \frac{\omega}{q} q_{t}^{2},
\end{aligned}
$$

where $\omega_{i}=\left(4 \pi e^{2} n_{i} / m_{i}^{*}\right)^{1 / 2}$ is the plasma frequency of particles $i$, and $m_{i}^{*}$ is the effective particle mass at the appropriate Fermi surface. We have already defined $m_{e}^{*}$ and $m_{\mu}^{*}$ for electrons and muons which form almost ideal Fermi gases. Their effective masses differ from the bare masses owing to relativistic effects. As for protons $(i=p)$, which are nonrelativistic in neutron star cores, their effective mass $m_{p}^{*}$ differs from the bare proton mass due to strong interactions with surrounding nucleons. In the approximation (14) and (15) one can also neglect $\omega^{2}$ in the denominator of the second term in (11). Equations (13) are strictly valid for Fermi gases, which is a good approximation for the electrons and muons, but the protons constitute a Fermi liquid. Fortunately, the asymptotic expressions (14) and (15), sufficient for our calculations, remain valid for the Fermi liquid. This is because a kinetic equation for quasiparticles in the Fermi liquid at low $\omega$ is similar to a familiar kinetic equation for Fermi gases (see, e.g., Ref. [23]).

In Eqs. (14) and (15) we have introduced $q_{l}$ and $q_{t}$ $\left[\mathrm{cm}^{-1}\right]$ defined as (in standard physical units)

$$
\begin{aligned}
q_{l}^{2} & =\frac{4 \alpha}{\pi} \sum_{i} \frac{c m_{i}^{*} p_{F i}}{\hbar^{2}} \\
q_{t}^{2} & =\frac{4 \alpha}{\pi} \sum_{i} \frac{m_{i}^{*} p_{F i} v_{F i}}{\hbar^{2}}=\frac{4 \alpha}{\pi} \sum_{i} \frac{p_{F i}^{2}}{\hbar^{2}} .
\end{aligned}
$$

Generally, we have $q_{t} \leq q_{l}$. If all charged particles were ultrarelativistic then $q_{t} \rightarrow q_{l}$. 
According to Eqs. (14) and (15), the screening functions for longitudinal and transverse plasmons are different. The screening via longitudinal plasmons (14) is described by a real (nondissipative) polarization function $\Pi_{l}=q_{l}^{2}$ which is equivalent to a Debye-like (ThomasFermi) plasma screening with the screening length $1 / q_{l}$. The screening of transverse currents is described by a purely imaginary (dissipative) function (15), corresponding to the Landau damping via collisionless absorption of transverse plasmons by plasma particles. Calculating the electron and muon thermal conductivity in neutron star cores, the authors of Refs. [13, 17] have neglected the Landau damping and erroneously used the same static Debye-like screening, $\Pi_{t}=q_{l}^{2}$, for transverse currents as for longitudinal ones. We will show that this approximation strongly overestimates the thermal conductivity of electrons and muons.

The squared matrix element (11) for collisions between heat carriers $c$ and scatterers $i$ can be written as

$$
\left|M_{c i}\right|^{2}=\frac{16 \pi^{2} \alpha^{2}}{m_{c}^{* 2} m_{i}^{* 2}} \varphi, \quad \varphi=\varphi_{\|}+\varphi_{\perp}+\varphi_{\perp \|}
$$

where $\varphi$ is a dimensionless function determined by the exchange of longitudinal plasmons $\left(\varphi_{\|}\right)$, transverse plasmons $\left(\varphi_{\perp}\right)$, and by both interactions $\left(\varphi_{\| \perp}\right)$. The leading terms in the weak screening approximation are

$$
\begin{aligned}
\varphi_{\|} & =\frac{m_{c}^{* 2} m_{i}^{* 2}}{\left(q^{2}+q_{l}^{2}\right)^{2}}, \\
\varphi_{\perp} & =\frac{p_{F c}^{2} p_{F i}^{2} q^{2} \cos ^{2} \phi}{q^{6}+\Lambda^{6}}, \\
\varphi_{\perp \|} & =-\frac{2 m_{c}^{*} m_{i}^{*} p_{F i} p_{F c} q^{4} \cos \phi}{\left(q^{2}+q_{l}^{2}\right)\left(q^{6}+\Lambda^{6}\right)}
\end{aligned}
$$

where $\Lambda=\left(\pi \omega q_{t}^{2} / 4\right)^{1 / 3}$ and $\phi$ is the angle between $\boldsymbol{p}_{1}$ and $\boldsymbol{p}_{2}$. For collisions of identical particles $(i=c)$, the function $\varphi$ is generally different but the difference is negligible in the leading-order weak screening approximation.

\section{B. Calculation of collision frequencies}

Calculating the integrals (8) for a strongly degenerate matter, we can use the standard angular-energy decomposition (e.g., Ref. 1]). It consists in placing momenta of colliding particles at respective Fermi surfaces, whenever possible, and in decoupling energy and angular integrations using $\mathrm{d}^{3} p=m^{*} p_{F} \mathrm{~d} \varepsilon \mathrm{d} \Omega$. The squared matrix element in (91) depends only on relative orientations of momenta of colliding particles which greatly simplifies angular integrations. Performing similar transformations as in Gnedin and Yakovlev [17] we can reduce Eqs. (7) and (8) for the collision frequencies of nonidentical par- ticles to

$$
\begin{aligned}
\nu_{c i} & =\frac{12 \alpha^{2}}{\pi^{4} T^{3} p_{F c} m_{c}^{*}}\left(J_{c i 1}-J_{c i 1^{\prime}}\right), \\
\nu_{c i}^{\prime} & =\frac{12 \alpha^{2} p_{F i}}{\pi^{4} T^{3} p_{F c}^{2} m_{i}^{*}}\left(J_{c i 2}-J_{c i 2^{\prime}}\right) .
\end{aligned}
$$

Here,

$$
\begin{aligned}
J_{c i k}= & \iiint \int \mathrm{d} \varepsilon_{1} \mathrm{~d} \varepsilon_{1}^{\prime} \mathrm{d} \varepsilon_{2} \mathrm{~d} \varepsilon_{2}^{\prime} f_{1} f_{2}\left(1-f_{1}^{\prime}\right)\left(1-f_{2}^{\prime}\right) \\
& \times\left(\varepsilon_{1}-\mu_{1}\right)\left(\varepsilon_{k}-\mu_{k}\right) I_{\Omega k}(\omega)
\end{aligned}
$$

is an energy integral and $I_{\Omega k}(\omega)$ is a remaining angular integral, which depends on a collision energy transfer $\omega$ owing to the dynamical screening in Eq. (15). The effective collision frequency $\nu_{c c}$ between identical heat carriers can be calculated as $\nu_{c c}=\nu_{c i}+\nu_{c i}^{\prime}$ with $i=c$.

In the weak screening approximation we obtain

$$
\begin{aligned}
& I_{\Omega 1}=I_{\Omega 1^{\prime}}=\int_{0}^{q_{m}} \mathrm{~d} q \int_{0}^{\pi} \mathrm{d} \phi \varphi, \\
& I_{\Omega 2}=I_{\Omega 2^{\prime}}=\int_{0}^{q_{m}} \mathrm{~d} q \int_{0}^{\pi} \mathrm{d} \phi \varphi \cos \phi,
\end{aligned}
$$

with $q_{m}=\min \left\{2 p_{F i}, 2 p_{F c}\right\}$. The integration over $\phi$ is then trivial. From Eqs. (19) -(21) we see that $I_{\Omega 1}$ consists of two terms associated with the longitudinal and transverse plasmon exchange, respectively, while $I_{\Omega 2}$ contains only the mixed term,

$$
I_{\Omega 1}=I_{\Omega 1}^{\perp}+I_{\Omega 1}^{\|}, \quad I_{\Omega 2}=I_{\Omega 2}^{\perp \|} .
$$

This means, that $\nu_{c i}$ is determined by the contributions from longitudinal and transverse screening, but $\nu_{c i}^{\prime}$ is determined by the mixed term.

We have calculated the angular integrals in the weak screening approximation, retaining the leading terms in series expansions over $q_{l}$ or $\Lambda$. The result is

$$
\begin{aligned}
I_{\Omega 1}^{\perp} & =\frac{\pi}{3} \frac{p_{F i}^{2} p_{F c}^{2}}{|\omega|} \frac{1}{q_{t}^{2}} \\
I_{\Omega 1}^{\|} & =\frac{\pi^{2} m_{c}^{* 2} m_{i}^{* 2}}{4 q_{l}^{3}} \\
I_{\Omega 2}^{\perp \|} & =-\frac{\pi^{2}}{3}\left(\frac{4}{\pi}\right)^{1 / 3} \frac{p_{F i} p_{F c}}{q_{l}^{2}} \frac{m_{i}^{*} m_{c}^{*}}{q_{t}^{2 / 3}|\omega|^{1 / 3}} .
\end{aligned}
$$

Using the standard technique (e.g., [17]) we can reduce the energy integration to

$$
\begin{aligned}
& J_{c i 1}-J_{c i 1^{\prime}}=\int_{0}^{\infty} \frac{I_{\Omega 1}(\omega) \omega^{3} \exp (-\omega / T) \mathrm{d} \omega}{[1-\exp (-\omega / T)]^{2}} \\
& J_{c i 2}-J_{c i 2^{\prime}}=-\int_{0}^{\infty} \frac{I_{\Omega 2}(\omega) \omega^{3} \exp (-\omega / T) \mathrm{d} \omega}{[1-\exp (-\omega / T)]^{2}}
\end{aligned}
$$


The final $\omega$ integration gives analytical expressions for the collision frequencies (in standard physical units)

$$
\begin{aligned}
\nu_{c i} & =\nu_{c i}^{\perp}+\nu_{c i}^{\|}, \\
\nu_{c i}^{\perp} & =\frac{24 \zeta(3)}{\pi^{3}} \frac{\alpha^{2} k_{B} T}{\hbar c m_{c}^{*}} \frac{p_{F i}^{2} p_{F c}}{\hbar^{2} q_{t}^{2}}, \\
\nu_{c i}^{\|} & =\frac{4 \pi^{2}}{5} \frac{\alpha^{2} k_{B}^{2} T^{2}}{\hbar c^{2} m_{c}^{*}} \frac{c^{4} m_{c}^{* 2} m_{i}^{* 2}}{p_{F c} \hbar^{3} q_{l}^{3}}, \\
\nu_{c i}^{\prime} & =\frac{12 \xi}{\pi^{3}} \frac{\alpha^{2}\left(k_{B} T\right)^{5 / 3}}{\hbar\left(\hbar c q_{t}\right)^{2 / 3}} \frac{c m_{c}^{*} p_{F i}^{2}}{\hbar^{2} p_{F c} q_{l}^{2}}, \\
\xi & =\frac{(2 \pi)^{2 / 3}}{3} \Gamma\left(\frac{14}{3}\right) \zeta\left(\frac{11}{3}\right) \approx 18.52,
\end{aligned}
$$

where $\zeta(z)$ is the Riemann zeta function, and $\Gamma(z)$ is the gamma function. Equations (4) - (6) and (33)-(36) fully determine the electron and muon thermal conductivity of a nonsuperconducting matter calculated in the weak screening approximation.

Owing to a strong degeneracy, for typical conditions in neutron star cores

$$
\nu_{c i}^{\|} \ll \nu_{c i}^{\prime} \ll \nu_{c i}^{\perp} .
$$

Because $\nu_{e \mu}^{\prime}$ and $\nu_{\mu e}^{\prime}$ are negligible, electron and muon heat transports $(c=e$ and $\mu$ ) become formally decoupled. In this approximation from Eq. (5) we obtain

$$
\frac{1}{\tau_{c}}=\nu_{c}^{\perp}=\sum_{i} \nu_{c i}^{\perp}=\frac{6 \zeta(3)}{\pi^{2}} \frac{\alpha k_{B} T p_{F c}}{\hbar c m_{c}^{*}} .
$$

Here the electron and muon thermal relaxation times $\tau_{e}$ and $\tau_{\mu}$ are mostly determined by the exchange of transverse plasmons (by the Landau damping) and are expressed only through the parameters of heat carriers $(e$ or $\mu$ ).

The thermal conductivity of the electrons or muons becomes temperature independent,

$$
\kappa_{c}=\kappa_{c}^{\perp}=\frac{\pi^{2}}{54 \zeta(3)} \frac{k_{B} c p_{F c}^{2}}{\hbar^{2} \alpha},
$$

being solely determined by the Fermi momentum $p_{F c}$ of heat carriers.

The remarkable simplicity of the thermal conductivity of strongly degenerate particles in this regime, where electromagnetic interactions are dominated by the Landau damping of transverse plasmons, was pointed out by Pethick and Heiselberg [18] for a plasma of massless (ultrarelativistic) quarks. The authors stressed that the temperature dependence of this thermal conductivity strongly deviates from the dependence $\kappa \propto T^{-1}$ in an ordinary Fermi liquid, because the plasma screening due to the exchange of transverse plasmons depends on energy transfer $\hbar \omega$ in collision events. Were particle collisions dominated by the exchange of longitudinal plasmons $\left(\nu=\nu^{\|}\right)$, the thermal conductivity would show the traditional Fermi-liquid behavior (as was obtained in
Refs. [13, 16, 17], whose authors erroneously assumed the Debye-like plasma screening, the same as determined by the exchange of longitudinal plasmons).

Recently we [19] have calculated $\nu_{e e}$, taking proper account of the electron plasma screening via the exchange of longitudinal and transverse plasmons, for degenerate electrons of any degree of relativity. We have used more general electron polarization functions given by Eqs. (12) and (13) ) rather than by the asymptotic limits (14) and (15) employed in the present paper]. We have applied our results for studying the electron thermal conductivity in a neutron star crust, but after adding the plasma screening by muons and protons [see Eqs. (16) and (17)] they become valid for nonsuperconducting neutron star cores. Replacing in $\nu_{e e}$ electrons by muons we can obtain the expression for $\nu_{\mu \mu}$.

Our present results include electromagnetic interactions of electrons, muons and protons (not only $\nu_{e e}$ or $\nu_{\mu \mu}$ which could be extracted from the results of Ref. [19]); they are valid for degenerate particles of any degree of relativity. Because the exchange of transverse plasmons is efficient for relativistic particles, it becomes unimportant if all charged particles are nonrelativistic. The presence of ultrarelativistic electrons in neutron star cores ensures the importance of the Landau damping there.

\section{Comparison with exact solution}

So far we have used a simplest variational thermal conductivity based on the approximation (3) with $\tau_{c}$ independent of $\varepsilon_{c}$. However, the actual energy dependence of the function $\Phi_{c}$ in the exact solution is more complicated. As already mentioned above, Gnedin and Yakovlev 17. introduced a factor $C$ which corrects the variational thermal conductivity, $\kappa_{\text {exact }}=C \kappa_{\text {var }}$. For the electromagnetic interaction wholly (and erroneously) attributed to the exchange of longitudinal plasmons, in the weakscreening approximation they obtained $C \approx 1.2-1.3$ and suggested to use $C=1.2$. Their calculation was based on the exact transport theory in Fermi liquids developed by Sykes and Brooker [24] for one-component systems and extended for multi-component systems by Flowers and Itoh [13] and Anderson et al. [25]. Notice that if Gnedin and Yakovlev correctly calculated the thermal conductivity solely determined by the exchange of longitudinal plasmons they would obtain the parameter $\lambda$ from their Eq. (62) equal to 1 and $C=1.2$. The exact theory [24] cannot be directly applied to our case because the Landau damping of transverse plasmons makes the matrix element $\omega$-dependent, that is not characteristic for ordinary Fermi liquids.

In this section we estimate $C$ taking into account only the exchange of transverse plasmons in the weak screening approximation for one type of heat carriers (recall that the electron and muon heat transports become decoupled). We use the straightforward generalization of the exact theory [13, 24, 25, 26]. Instead of Eq. (3) we 
write

$$
\Phi_{c}=-\tau_{\mathrm{eff}} \Psi(x) \boldsymbol{v}_{c} \cdot \nabla T,
$$

where $\tau_{\text {eff }}$ is an effective relaxation time, while $\Psi(x)$ is an unknown function of $x=\left(\varepsilon-\mu_{c}\right) / T$. Following Ref. [26] we substitute (41) into the linearized kinetic equation and obtain the equation for $\Psi(x)$,

$$
\begin{aligned}
- & \frac{x}{1+\exp (x)} \\
& =\int_{-\infty}^{\infty} \mathrm{d} x_{1} \frac{\operatorname{sign}\left(x_{1}-x\right)}{1+\exp \left(x_{1}\right)} \frac{\Psi\left(x_{1}\right)-\Psi(x)}{1-\exp \left(x-x_{1}\right)},
\end{aligned}
$$

with $\tau_{\text {eff }}=3 /\left(\alpha v_{F c} T\right)$, and the thermal conductivity calculated as

$$
\kappa_{\text {exact }}=\frac{n_{c} \tau_{\text {eff }}}{m_{c}^{*}} \int_{-\infty}^{\infty} \mathrm{d} x x \Psi(x) f_{c}\left(1-f_{c}\right) .
$$

We have solved Eq. (42) numerically, calculated $\kappa_{\text {exact }}$, and compared it with the variational result (that corresponds to $\left.\Psi_{\mathrm{var}}(x)=\pi^{2} x /[18 \zeta(3)]\right)$. The difference between the exact and variational results appears to be negligible, $C=\kappa_{\text {exact }} / \kappa_{\text {var }} \approx 1$. In a more general case the electron (muon) scattering is determined by the exchange of both transverse and longitudinal plasmons and we can expect that $1 \leq C \leq 1.2$.

\section{EFFECTS OF PROTON SUPERCONDUCTIVITY}

In the previous sections we have considered nonsuperconducting protons. However, it is well known that the protons in neutron star cores can be in a superfluid (superconducting) state (e.g., Lombardo and Schulze [27]). Let us include the effects of proton superconductivity.

Microscopically, the onset of superconductivity after the temperature $T$ falls below a (density dependent) critical temperature $T_{c p}$ manifests itself in the appearance of a gap $\Delta=\Delta(T)$ in the proton energy spectrum in the vicinity of the proton Fermi level. Instead of protons, it is now necessary to introduce Bogoliubov quasiparticles with energies (with respect to the Fermi level $\mu_{p}$ ),

$$
\epsilon-\mu_{p}=\operatorname{sign}\left(p-p_{F p}\right) \sqrt{\Delta^{2}+v_{F p}^{2}\left(p-p_{F p}\right)^{2}} .
$$

It is generally believed, that proton pairing in neutron star cores occurs in the ${ }^{1} S_{0}$ channel. In this case, the gap is isotropic (independent of orientation of proton momentum with respect to a quantization axis). For the Bardeen-Cooper-Schrieffer (BCS) pairing model the temperature dependence of $\Delta$ at $0 \leq T \leq T_{c p}$ can be fitted as 28]

$$
y \equiv \frac{\Delta}{k_{B} T}=\sqrt{1-t}\left(1.456-\frac{0.157}{\sqrt{t}}+\frac{1.764}{t}\right),
$$

where $t=T / T_{c p}$.

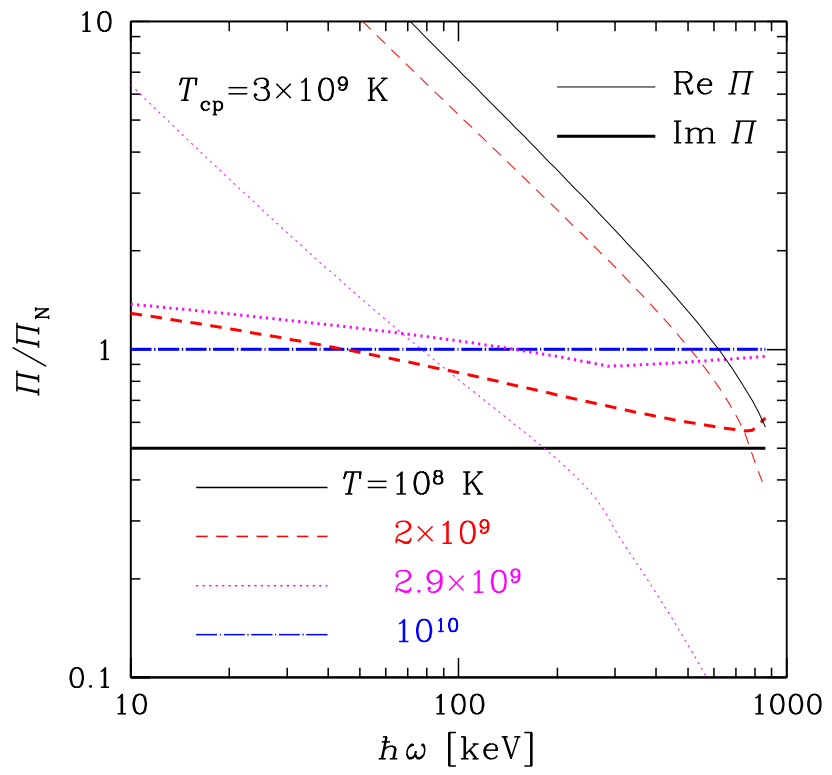

Figure 1: (Color online) Real and imaginary parts (thin and thick lines, respectively) of the proton transverse polarization function $\Pi_{t}^{(p)}$ versus $\hbar \omega$ at small $q$ in superconducting matter for the proton critical temperature $T_{c p}=3 \times 10^{9} \mathrm{~K}$ and four values of the temperature, $T=10^{8} \mathrm{~K}$ (solid lines), $2 \times 10^{9} \mathrm{~K}$ (dashed lines), $2.9 \times 10^{9} \mathrm{~K}$ (dotted lines), and $10^{10}$ $\mathrm{K}$ (dot-dashed lines). The plotted functions are divided by the imaginary part of $\Pi_{t \mathrm{~N}}^{(p)}$ in normal matter.

\section{A. Dielectric function}

Proton superconductivity affects electrodynamical properties of dense matter. The longitudinal polarization function $\Pi_{l}$ undergoes no significant changes (see, e.g., Ref. [29]; a strong effect of superconductivity on longitudinal plasma screening described by the function $Z$ in Eq. (47) of Ref. 17] is in error; one should set $Z=1$ in equations of Ref. [17]). In contrast, the proton component $\Pi_{t}^{(p)}$ of the total transverse function $\Pi_{t}=\Pi_{t}^{(e)}+\Pi_{t}^{(\mu)}+\Pi_{t}^{(p)}$ is considerably modified. It should be stressed that we discuss the electromagnetic polarization functions to be distinguished from polarization functions associated with strong interactions (e.g., Ref. [30]).

For our purpose, we employ the model of a Fermi gas of protons with BCS superconductivity in the so called Pippard limit appropriate to neutron star cores $(\hbar \omega \ll$ $p_{F p} v_{F p}, \hbar q \ll p_{F p}$, but $\xi q \gg 1$, with $\xi \sim \hbar v_{F p} / k_{B} T_{c p}$ being the coherence length). The polarization functions appropriate to this case are well known. They were calculated in the classical paper by Mattis and Bardeen [31]. In the limit of small $\omega$ and $q$, we are interested in, these results are gauge invariant [29]. The effects of strong (nuclear) interactions in the proton Fermi liquid (particularly, dragging of neutrons by protons) on the electromagnetic polarization functions are complicated and almost 
not explored; they are beyond the scope of the present paper. According to M. E. Gusakov (private communication) these effects should not greatly modify our results. Similar effects of strong interactions on the polarization functions of superfluid neutrons [30] lead to renormalization of vertices and take into account specific collective superfluid excitations. These effects can be pronounced for those $q$ and $\omega$ which are typical of such excitations $\left(\omega \sim q v_{F}\right)$, and the effects are small outside such $q-\omega$ domains. We expect that in our case $\left(\omega \ll q v_{F}\right)$ these effects are small and can be neglected.

Therefore, using the classical polarization functions [31] seems to be a good starting approximation. It gives

$$
\Pi_{t}^{(p)}=\frac{q_{t}^{2}}{4} \frac{\Delta}{q} Q
$$

where $Q$ is a complex function of the normalized frequency $\tilde{\omega}=\hbar \omega / \Delta$ and the normalized gap $y$ (or, alternatively, of $\hbar \omega / k_{B} T$ and $y$ ). The real and imaginary parts of $Q$ are given by

$$
\begin{aligned}
\Re Q= & \pi \int_{\max [\tilde{\omega}-1,1]}^{\tilde{\omega}+1} \tanh \left(\frac{y}{2} E\right) \\
& \times \frac{[E(E-\tilde{\omega})+1] \mathrm{d} E}{\sqrt{\left(E^{2}-1\right)\left(1-(E-\tilde{\omega})^{2}\right)}}, \\
\Im Q= & \pi \int_{1}^{\infty}\left\{\tanh \left(\frac{y}{2}(E+\tilde{\omega})\right)-\tanh \left(\frac{y}{2} E\right)\right\} \\
& \times \frac{[E(E+\tilde{\omega})+1] \mathrm{d} E}{\sqrt{\left(E^{2}-1\right)\left((E+\tilde{\omega})^{2}-1\right)}} \\
+ & \pi \Theta(\tilde{\omega}-2) \int_{1}^{\tilde{\omega}-1} \tanh \left(\frac{y}{2} E\right) \\
& \times \frac{[E(\tilde{\omega}-E)-1] \mathrm{d} E}{\sqrt{\left(E^{2}-1\right)\left((\tilde{\omega}-E)^{2}-1\right)}},
\end{aligned}
$$

where $\Theta(x)=0$ at $x \leq 0$ and $\Theta(x)=1$ otherwise. Here and below we again return to units $c=\hbar=k_{B}=1$ unless the contrary is indicated.

In the nonsuperconding limit, $y \rightarrow 0$, only the imaginary part of $Q$ survives, giving $Q=i \pi \tilde{\omega}$ and reproducing the asymptote (15) determined by the Landau damping (dissipative plasma screening). In the opposite limit of strong superconductivity, $y \rightarrow \infty$, the imaginary part of $Q$ is small (because the gap in the proton energy spectrum becomes much larger than characteristic values of $\left.\hbar \omega \sim k_{B} T\right)$. Then only nondissipative plasma screening is available (with $Q=\pi^{2}$ ). These results are illustrated in Fig. 1 which shows the frequency dependence of the real and imaginary parts of $\Pi_{t}^{(p)}$ in superconducting matter. The presented functions are normalized by the imaginary (Landau damping) part of $\Pi_{t}^{(p)}$ in normal matter. The critical temperature of proton superconductivity is taken to be $T_{c p}=3 \times 10^{9} \mathrm{~K}\left(k_{B} T_{c p}=259 \mathrm{keV}\right)$. The frequency dependence is presented for four values of the temperature $T$. The highest temperature $T=10^{10} \mathrm{~K}$ $\left(k_{B} T=862 \mathrm{keV}\right)$ refers to nonsuperconducting matter $(y=0, \Delta=0)$. The proton polarization is provided by the Landau damping. The displayed normalized polarization function has the imaginary Landau-damping part (the thick dot-dashed line which is the reference line of the figure); the real part is essentially zero. The dotted, dash-dot, and solid lines refer to progressively lower $T=2.9 \times 10^{9}, 2 \times 10^{9}$, and $10^{8} \mathrm{~K}\left(k_{B} T=250,172\right.$, and $8.62 \mathrm{keV}$ ) in superconducting matter (with $y=0.570$, 2.26 , and 52.6 ; and $\Delta=142,389$, and $453 \mathrm{keV}$, respectively). One can observe the growth of the real part of the normalized polarization function, which becomes larger than the imaginary part, leading to a nondissipative transverse plasma screening for $T \ll T_{c p}$.

The total transverse polarization function of the plasma containing electrons, muons and superconducting protons has the form

$$
\begin{aligned}
\Pi_{t} & =\frac{\pi \omega}{4 q}\left\{q_{t p}^{2} \frac{\Delta}{\pi \omega} \Re Q\right. \\
& \left.+i\left(q_{t e}^{2}+q_{t \mu}^{2}+q_{t p}^{2} \frac{\Delta}{\pi \omega} \Im Q\right)\right\},
\end{aligned}
$$

where $q_{t i}^{2}=4 \alpha p_{F i}^{2} / \pi$. In the case of strong superconductivity, the main contribution to transverse screening comes from superconducting protons. When $T$ decreases from $T_{c p}$ to zero, the transverse plasma screening turns from dissipative to nondissipative one.

Thus, proton superconductivity modifies the transverse polarization function and the screening functions (20) and (21). One can show that in the weak screening approximation the angular integrals in superconducting matter can be presented as

$$
\begin{aligned}
I_{\Omega 1}^{\perp S} & =I_{\Omega 1}^{\perp} F^{\perp}(\omega / T, y, r), \\
I_{\Omega 2}^{\perp \| S} & =I_{\Omega 2}^{\perp \|} F^{\perp \|}(\omega / T, y, r),
\end{aligned}
$$

where $I_{\Omega 1}^{\perp}$ and $I_{\Omega 2}^{\perp \|}$ are the angular integrals in the nonsuperconducting matter, while

$$
\begin{aligned}
F^{\perp}= & \frac{\pi|\omega|(r+1)}{|\pi \omega r+\Delta \Im Q|} \\
& \times\left(1-\frac{2}{\pi} \arctan \frac{\Delta \Re Q}{|\pi \omega r+\Delta \Im Q|}\right), \\
F^{\| \perp}= & \frac{[\pi|\omega|(r+1)]^{1 / 3}\left[(\pi \omega r+\Delta \Im Q)^{2}+(\Delta \Re Q)^{2}\right]^{1 / 3}}{|\pi \omega r+\Delta \Im Q|} \\
\times & \frac{2}{\sqrt{3}} \sin \left(\frac{2}{3} \arctan \frac{|\pi \omega r+\Delta \Im Q|}{\Delta \Re Q}\right) \\
+ & \frac{[\pi|\omega|(r+1)]^{1 / 3} \Delta \Re Q}{|\pi \omega r+\Delta \Im Q|\left[(\pi \omega r+\Delta \Im Q)^{2}+(\Delta \Re Q)^{2}\right]^{1 / 6}} \\
\times & \frac{2}{\sqrt{3}} \sin \left(\frac{1}{3} \arctan \frac{|\pi \omega r+\Delta \Im Q|}{\Delta \Re Q}\right)
\end{aligned}
$$


are the factors which take into account the effects of superconductivity. The parameter $r$ is defined as

$$
r=\frac{q_{t e}^{2}+q_{t \mu}^{2}}{q_{t p}^{2}}=\frac{p_{F e}^{2}+p_{F \mu}^{2}}{p_{F p}^{2}} .
$$

It is a slowly variable function of the density in a neutron star core, constrained by the condition of plasma neutrality, $n_{e}+n_{\mu}=n_{p}$. We have $r=1$ in the absence of muons, and $r>1$ if the muons are present. The maximum value $r_{\max }=2^{1 / 3} \approx 1.26$ is reached in the high-density limit in which the muons become ultrarelativistic.

In the limiting case of strong superconductivity $(y \gg$ 1)

$$
\begin{aligned}
F^{\perp} & =\frac{2|\omega|(r+1)}{\pi^{2} \Delta}, \\
F^{\| \perp} & =\frac{2}{\sqrt{3}}\left(\frac{|\omega|(r+1)}{\pi \Delta}\right)^{1 / 3} .
\end{aligned}
$$

The collision frequencies involving nonsuperconducting particles $(e e, \mu \mu$, and $e \mu$ ) can be calculated directly from Eqs. (31) and (32), taking into account the factors $F^{\perp}$ and $F^{\| \perp}$. It is convenient to present these collision frequencies in the form

$$
\begin{aligned}
\nu_{c i}^{\perp S} & =\nu_{c i}^{\perp} R_{\ell}^{\perp}(y, r), \\
\nu_{c i}^{\prime S} & =\nu_{c i}^{\prime} R_{\ell}^{\| \perp}(y, r),
\end{aligned}
$$

where $\nu_{c i}^{\perp}$ and $\nu_{c i}^{\prime}$ correspond to nonsuperconducting matter, while

$$
\begin{aligned}
R_{\ell}^{\perp}(y, r)= & \frac{1}{6 \zeta(3)} \int_{0}^{\infty} \frac{\omega^{3} \exp (-\omega / T) F^{\perp} \mathrm{d} \omega}{[1-\exp (-\omega / T)]^{2}}, \\
R_{\ell}^{\| \perp}(y, r)= & \frac{1}{\Gamma(14 / 3) \zeta(11 / 3)} \\
& \times \int_{0}^{\infty} \frac{\omega^{11 / 3} \exp (-\omega / T) F^{\| \perp} \mathrm{d} \omega}{[1-\exp (-\omega / T)]^{2}}
\end{aligned}
$$

describe the effect of proton superconductivity. In the case of strong superconductivity $(y \gg 1)$ we obtain the asymptotes

$$
\begin{aligned}
R_{\ell}^{\perp}(y, r) & =\frac{4 \pi^{2}(r+1)}{45 \zeta(3) y} \\
R_{\ell}^{\| \perp}(y, r) & =\frac{8 \pi^{4}}{\Gamma(14 / 3) \zeta(11 / 3) 15 \sqrt{3}}\left(\frac{r+1}{\pi y}\right)^{1 / 3} .
\end{aligned}
$$

We have calculated $R_{\ell}^{\| \perp}(y, r)$ on a dense grid of $y$ and $r$ and fitted the results by the function

$$
R_{\ell}^{\| \perp}(y, r)=\frac{(r+1)^{1 / 3}}{\left[(r+1)^{2}-0.757 y+(0.50651 y)^{2}\right]^{1 / 6}} .
$$

The fit reproduces numerical data, the above asymptote at $y \rightarrow \infty$, and the evident condition $R_{\ell}^{\| \perp}(0, r)=1$. The maximum relative fit error $\approx 8 \%$ occurs at $y=2.2667$ and $r=1.3$. We do not present any separate fit formula for $R_{\ell}^{\perp}(y, r)$ but obtain a practical expression for $\nu_{c}^{\perp S}$ in the next section.

\section{B. Superconducting scatterers}

Aside of changing plasma screening, proton superconductivity directly affects the partial collision frequencies $\nu_{e p}$ and $\nu_{\mu p}$. Now these collision frequencies describe interaction of electrons or muons with proton Bogoliubov quasiparticles. Because the number of such quasiparticles is not necessarily conserved, the collision frequencies should include contributions of three processes, (1) scattering $\left(1,2 \rightarrow 1^{\prime}, 2^{\prime}\right) ;(2)$ decay $\left(1 \rightarrow 1^{\prime},-2,2^{\prime}\right)$; and (3) coalescence $\left(1,2,-2^{\prime} \rightarrow 1^{\prime}\right)$. Here, 1 and $1^{\prime}$ refer to an electron or muon; $2,2^{\prime},-2$, and $-2^{\prime}$ refer to Bogoliubov proton quasiparticles; -2 (or $-2^{\prime}$ ) stands for a proton quasiparticle with momentum and spin directions opposite to those for 2 (or $\left.2^{\prime}\right)$. A linearized collision integral $I_{c p}$ becomes

$$
I_{c p}=I_{c p}^{\mathrm{sc}}+I_{c p}^{\mathrm{dec}}+I_{c p}^{\mathrm{coal}}
$$

where

$$
\begin{aligned}
I_{c p}^{\mathrm{sc}}= & \int \mathrm{d} \Gamma f_{1} f_{2}\left(1-f_{1^{\prime}}\right)\left(1-f_{2^{\prime}}\right) \\
& \times W_{c p}^{\mathrm{sc}}\left(1,2 \mid 1^{\prime}, 2^{\prime}\right)\left(\Phi_{1^{\prime}}-\Phi_{1}\right), \\
I_{c p}^{\mathrm{dec}}= & \frac{1}{2} \int \mathrm{d} \Gamma f_{1}\left(1-f_{-2}\right)\left(1-f_{1^{\prime}}\right)\left(1-f_{2^{\prime}}\right) \\
& \times W_{c p}^{\mathrm{dec}}\left(1 \mid 1^{\prime},-2,2^{\prime}\right)\left(\Phi_{1^{\prime}}-\Phi_{1}\right), \\
I_{c p}^{\mathrm{coal}}= & \frac{1}{2} \int \mathrm{d} \Gamma f_{1} f_{2} f_{-2^{\prime}}\left(1-f_{1^{\prime}}\right) \\
& \times W_{c p}^{\mathrm{coal}}\left(1,2,-2^{\prime} \mid 1^{\prime}\right)\left(\Phi_{1^{\prime}}-\Phi_{1}\right),
\end{aligned}
$$

correspond to scattering (sc), decay (dec), and coalescence (coal), respectively; $\mathrm{d} \Gamma=(2 \pi \hbar)^{-9} \mathrm{~d}^{3} p_{2} \mathrm{~d}^{3} p_{1^{\prime}} \mathrm{d}^{3} p_{2^{\prime}}$. The factor $\frac{1}{2}$ excludes double counting of the same collision events.

Let us focus on the differential transition probabilities $\quad W_{c p}^{\mathrm{sc}}\left(1,2 \mid 1^{\prime}, 2^{\prime}\right), \quad W_{c p}^{\mathrm{dec}}\left(1 \mid 1^{\prime},-2,2^{\prime}\right), \quad$ and $W_{c p}^{\text {coal }}\left(1,2,-2^{\prime} \mid 1^{\prime}\right)$. In the case of scattering, according to Fermi Golden Rule,

$$
W_{c p}^{\mathrm{sc}}=2 \pi \delta\left(\varepsilon_{1}+\varepsilon_{2}-\varepsilon_{1^{\prime}}-\varepsilon_{2^{\prime}}\right) \sum_{\text {spins }}\left|\left\langle 1^{\prime}, 2^{\prime}|\hat{V}| 1,2\right\rangle\right|^{2},
$$

where

$$
\begin{aligned}
\hat{V}= & \alpha \iint \mathrm{d} \boldsymbol{r}_{1} \mathrm{~d} \boldsymbol{r}_{2}\left[\hat{\rho}_{c}\left(\boldsymbol{r}_{1}\right) \hat{\rho}_{p}\left(\boldsymbol{r}_{2}\right) D_{\|}\left(\boldsymbol{r}_{1}-\boldsymbol{r}_{2}\right)\right. \\
& \left.-\left(\hat{\boldsymbol{J}}_{c}\left(\boldsymbol{r}_{1}\right) \cdot \hat{\boldsymbol{J}}_{p}\left(\boldsymbol{r}_{2}\right)\right) D_{\perp}\left(\boldsymbol{r}_{1}-\boldsymbol{r}_{2}\right)\right]
\end{aligned}
$$

is the interaction operator, $D_{\|}$and $D_{\perp}$ being corresponding propagators. In the first approximation, protons in neutron star cores can be treated as nonrelativistic particles. Then the second-quantized operators of the proton density and proton current are

$$
\begin{aligned}
& \hat{\rho}_{p}=\hat{\psi}^{\dagger} \hat{\psi}, \\
& \hat{\boldsymbol{J}}_{p}=\frac{i}{2 m_{p}^{*}}\left[\left(\boldsymbol{\nabla} \hat{\psi}^{\dagger}\right) \hat{\psi}-\hat{\psi}^{\dagger} \boldsymbol{\nabla} \hat{\psi}\right] .
\end{aligned}
$$


The psi-operator of proton field is

$$
\hat{\psi}=\sum_{\boldsymbol{p} \sigma} \chi_{\sigma} \exp (i \boldsymbol{p} \cdot \boldsymbol{r}) \hat{a}_{\boldsymbol{p} \sigma},
$$

where $\hat{a}_{\boldsymbol{p} \sigma}$ is a proton (not a Bogoliubov quasi-particle) annihilation operator and $\chi_{\sigma}$ is a unit basic spinor. Bogoliubov quasiparticles are introduced through quasiparticle annihilation operators $\hat{b}_{\boldsymbol{p} \sigma}$ basing on the Bogoliubov transformation

$$
\begin{aligned}
& \hat{b}_{\boldsymbol{p} \sigma}=\mathrm{u}_{p} \hat{a}_{\boldsymbol{p} \sigma}-\operatorname{sign}(\sigma) \mathrm{v}_{p} \hat{a}_{-\boldsymbol{p},-\sigma}^{\dagger}, \\
& \hat{a}_{\boldsymbol{p} \sigma}=\mathrm{u}_{p} \hat{b}_{\boldsymbol{p} \sigma}+\operatorname{sign}(\sigma) \mathrm{v}_{p} \hat{b}_{-\boldsymbol{p},-\sigma}^{\dagger},
\end{aligned}
$$

where $\mathrm{u}_{p}$ and $\mathrm{v}_{p}$ are coherence factors which can be chosen in different ways. We will use the following set of these factors

$$
\begin{aligned}
& \mathrm{u}_{p}=\frac{1}{\sqrt{2}} \sqrt{1+\frac{x}{z}}, \\
& \mathrm{v}_{p}=\frac{\operatorname{sign}(x)}{\sqrt{2}} \sqrt{1-\frac{x}{z}},
\end{aligned}
$$

where $x=v_{F p}\left(p-p_{F p}\right) / T$ and $z=\left(\varepsilon-\mu_{p}\right) / T$. In the nonsuperconducting limit we have $\mathrm{u}_{p}(y=0)=1$ and $\mathrm{v}_{p}(y=0)=0$. With this choice the dimensionless energy spectrum of quasiparticles is

$$
z=\frac{\varepsilon-\mu_{p}}{T}=\operatorname{sign}(x) \sqrt{x^{2}+y^{2}} .
$$

Obviously, the introduction of proton quasiparticles affects only the proton part of the matrix element that describes electromagnetic interaction. For the longitudinal part we have

$$
\left\langle 2^{\prime}\left|\hat{\rho}_{p}\right| 2\right\rangle=\left(\mathrm{u}_{2^{\prime}} \mathrm{u}_{2}-\mathrm{v}_{2^{\prime}} \mathrm{v}_{2}\right) \delta_{\sigma_{2} \sigma_{2^{\prime}}} \exp \left(i\left(\boldsymbol{p}_{2^{\prime}}-\boldsymbol{p}_{2}\right) \cdot \boldsymbol{r}\right) .
$$

It is clear that the longitudinal component of the matrix element $M_{\|}^{\text {sc }}$ differs from the same component $M_{\|}^{\text {norm }}$ in a nonsuperconducting (normal) case by

$$
M_{\|}^{\mathrm{sc}}=\left(\mathrm{u}_{2^{\prime}} \mathrm{u}_{2}-\mathrm{v}_{2^{\prime}} \mathrm{v}_{2}\right) M_{\|}^{\mathrm{norm}} .
$$

In the same way for the matrix element of the proton current $\left\langle 2^{\prime}\left|\hat{\boldsymbol{J}}_{p}\right| 2\right\rangle$, that describes the transverse interaction, we obtain

$$
M_{\perp}^{\mathrm{sc}}=\left(\mathrm{u}_{2^{\prime}} \mathrm{u}_{2}+\mathrm{v}_{2^{\prime}} \mathrm{v}_{2}\right) M_{\perp}^{\mathrm{norm}} .
$$

The differential transition probability for the decay channel has the form

$$
W_{c p}^{\mathrm{dec}}=2 \pi \delta\left(\varepsilon_{1}-\varepsilon_{2}-\varepsilon_{1^{\prime}}-\varepsilon_{2^{\prime}}\right) \sum_{\text {spins }}\left|\left\langle 1^{\prime},-2,2^{\prime}|\hat{V}| 1\right\rangle\right|^{2} .
$$

Notice that the energy conserving delta function differs from that in the scattering process. However, momentum and spin selection restrictions are the same for scattering, decay and coalescence processes because of the adopted choice of initial and final particle momenta and spin quantum numbers. We obtain

$$
\begin{aligned}
& M_{\|}^{\text {dec }}=\left(\mathrm{u}_{2^{\prime}} \mathrm{v}_{2}+\mathrm{u}_{2} \mathrm{v}_{2^{\prime}}\right) \operatorname{sign}\left(\sigma_{2}\right) M_{\|}^{\text {norm }}, \\
& M_{\perp}^{\text {dec }}=\left(\mathrm{u}_{2^{\prime}} \mathrm{v}_{2}-\mathrm{u}_{2} \mathrm{v}_{2^{\prime}}\right) \operatorname{sign}\left(\sigma_{2}\right) M_{\perp}^{\text {norm }} .
\end{aligned}
$$

Similarly, for the coalescence channel

$$
\begin{aligned}
W_{c p}^{\text {coal }}= & 2 \pi \delta\left(\varepsilon_{1}+\varepsilon_{2}-\varepsilon_{1^{\prime}}+\varepsilon_{2^{\prime}}\right) \\
& \times \sum_{\text {spins }}\left|\left\langle 1^{\prime}|\hat{V}| 1,2,-2^{\prime}\right\rangle\right|^{2}, \\
M_{\|}^{\text {coal }}= & \left(\mathrm{u}_{2^{\prime}} \mathrm{v}_{2}+\mathrm{u}_{2} \mathrm{v}_{2^{\prime}}\right) \operatorname{sign}\left(\sigma_{2}\right) M_{\|}^{\text {norm }}, \\
M_{\perp}^{\text {coal }}= & \left(\mathrm{u}_{2} \mathrm{v}_{2^{\prime}}-\mathrm{u}_{2^{\prime}} \mathrm{v}_{2}\right) \operatorname{sign}\left(\sigma_{2}\right) M_{\perp}^{\text {norm }} .
\end{aligned}
$$

Our procedure is standard and tacitly ignores renormalization of proton vertices in superconducting matter. It is justified because we are interested is small energy and momentum transfers $\omega$ and $q$ which are far from the transfers typical of collective superfluid excitations (see Refs. [29, 30] and Sec. [I A).

While integrating over quasiproton momenta in collision integrals we set $\mathrm{d}^{3} p=m_{p}^{*} T p_{F p} \mathrm{~d} x \mathrm{~d} \Omega$. It is instructive to replace $x_{2} \rightarrow-x_{2}$ for the decay case, and $x_{2^{\prime}} \rightarrow-x_{2^{\prime}}$ for coalescence. Such replacements change sign of quasiproton energy $\left(\varepsilon-\mu_{p}\right)$ and coherence factor $\mathrm{v}_{p}$ because of our choice of these factors. Then all energy-dependent terms, except for coherence factors, become the same in all three collision integrals. Hence we can describe the interaction of electrons (or muons) and quasiprotons by a unified collision integral (63), where $W_{c p}^{\text {sc }}\left(1,2 \mid 1^{\prime}, 2^{\prime}\right)$ is substituted by $W_{e p}^{S}\left(1,2 \mid 1^{\prime}, 2^{\prime}\right)$,

$$
\begin{aligned}
& W_{c p}^{S}\left(1,2 \mid 1^{\prime}, 2^{\prime}\right)=4(2 \pi)^{4} \delta\left(\varepsilon_{1}+\varepsilon_{2}-\varepsilon_{1}^{\prime}-\varepsilon_{2}^{\prime}\right) \\
& \quad \times \delta\left(\boldsymbol{p}_{1}+\boldsymbol{p}_{2}-\boldsymbol{p}_{1}^{\prime}-\boldsymbol{p}_{2}^{\prime}\right)\left|M_{c p}^{S}\right|^{2} \\
& \quad\left|M_{c p}^{S}\right|^{2}=\left|M_{c p}^{\mathrm{sc}}\right|^{2} \\
& \quad+\frac{1}{2}\left|M_{c p}^{\mathrm{dec}}\left(-x_{2}\right)\right|^{2}+\frac{1}{2}\left|M_{c p}^{\mathrm{coal}}\left(-x_{2}^{\prime}\right)\right|^{2} .
\end{aligned}
$$

Putting the squared matrix elements together, we have

$$
\begin{aligned}
\varphi_{\|}^{S} & =\left(1-4 \mathrm{u}_{2} \mathrm{u}_{2^{\prime}} \mathrm{v}_{2} \mathrm{v}_{2^{\prime}}\right) \varphi_{\|}, \\
\varphi_{\perp}^{S} & =\left(1+4 \mathrm{u}_{2} \mathrm{u}_{2^{\prime}} \mathrm{v}_{2} \mathrm{v}_{2^{\prime}}\right) \varphi_{\perp}, \\
\varphi_{\| \perp}^{S} & =\left(1-\mathrm{v}_{2}^{2}-\mathrm{v}_{2^{\prime}}^{2}\right) \varphi_{\| \perp},
\end{aligned}
$$

instead of $\varphi_{\|}, \varphi_{\perp}$, and $\varphi_{\| \perp}$ given by Eqs. (19)-(21) in the nonsuperconducting case.

Therefore, the expressions for $\nu_{e p}$ or $\nu_{\mu p}$ in the presence of proton superconductivity can be presented in the integral form similar to that in the nonsuperconducting case, but with two differences. First, coherence factors have to be introduced in the squared matrix element in accordance with Eq. (83). Second, proper quasiproton energies (73), containing energy gaps, should be used in Fermi-Dirac distribution functions of quasiprotons. Thus in order to calculate $\nu_{e p}$ and $\nu_{\mu p}$ we should reconsider not 
only the angular integration due to changes in polarization functions but the energy integration as well. The collision frequencies in question contain the transverse and longitudinal components (33). A careful examination of the derivation of the energy integral in (31) shows that for the collisions via the exchange of transverse plasmons one has

$$
\begin{aligned}
\nu_{c p}^{\perp S}= & \nu_{c p}^{\perp} R_{p}^{\perp}(y, r), \\
R_{p}^{\perp}(y, r)= & \frac{1}{6 \zeta(3)} \int_{0}^{\infty} \int_{0}^{\infty} \frac{\mathrm{d} x_{2} \mathrm{~d} x_{2}^{\prime}}{1+\exp \left(z_{2}\right)} \\
& \times\left\{\frac{\left(z_{2}^{\prime}-z_{2}\right)\left|z_{2}^{\prime}-z_{2}\right| F^{\perp}\left(\left|z_{2}^{\prime}-z_{2}\right|, y, r\right)}{\left[1+\exp \left(-z_{2}^{\prime}\right)\right]\left[\exp \left(z_{2}^{\prime}-z_{2}\right)-1\right]}\right. \\
& \times\left(1+4 \mathrm{u}_{2} \mathrm{u}_{2}^{\prime} \mathrm{v}_{2} \mathrm{v}_{2}^{\prime}\right) \\
& -\frac{\left(z_{2}^{\prime}+z_{2}\right)\left|z_{2}^{\prime}+z_{2}\right| F^{\perp}\left(\left|z_{2}^{\prime}+z_{2}\right|, y, r\right)}{\left[1+\exp \left(z_{2}^{\prime}\right)\right]\left[\exp \left(-z_{2}^{\prime}-z_{2}\right)-1\right]} \\
& \left.\times\left(1-4 \mathrm{u}_{2} \mathrm{u}_{2}^{\prime} \mathrm{v}_{2} \mathrm{v}_{2}^{\prime}\right)\right\}
\end{aligned}
$$

where $\nu_{c p}^{\perp}$ corresponds to normal protons, $R_{p}^{\perp}(y, r)$ describes the effects of superconductivity, $z_{2}=\left(\epsilon_{2}-\right.$ $\left.\mu_{p}\right) / k_{B} T=\sqrt{x_{2}^{2}+y^{2}}$ and $z_{2}^{\prime}=\left(\epsilon_{2}^{\prime}-\mu_{p}\right) / k_{B} T=$ $\sqrt{x_{2}^{\prime 2}+y^{2}}$.

In the limit of strong superconductivity $(y \gg 1)$ we have $R_{p}^{\perp}=A_{\perp}(r+1) \exp (-y)$, where

$$
\begin{aligned}
A_{\perp}= & \frac{4}{3 \pi^{2} \zeta(3)} \int_{0}^{\infty} \mathrm{d} \eta_{1} \int_{0}^{\infty} \mathrm{d} \eta_{2} \\
& \times \frac{\left(\eta_{1}^{2}-\eta_{2}^{2}\right)^{3}}{\exp \left(\eta_{1}^{2}\right)-\exp \left(\eta_{2}^{2}\right)} \approx 0.3446 .
\end{aligned}
$$

This strong superconductivity exponentially reduces the $e p$ and $\mu p$ collision rates. Nevertheless, the protons give the main contribution to the plasma polarization associated with the exchange of transverse plasmons. In this way they remain vitally important for the plasma screening in collisions involving leptons alone.

The total transverse collision frequency of heat carriers $c$ is

$$
\nu_{c}^{\perp S}=\sum_{i} \nu_{c i}^{\perp S}=\nu_{c}^{\perp} R_{\mathrm{tot}}^{\perp}(y, r)
$$

where $\nu_{c}^{\perp}$ is the total collision frequency in nonsuperconducting matter and

$$
R_{\mathrm{tot}}^{\perp}(y, r)=\frac{1}{r+1}\left[r R_{\ell}^{\perp}(y, r)+R_{p}^{\perp}(y, r)\right]
$$

describes the overall superconducting suppression of the total collision frequency via the exchange of transverse plasmons. In the limit of strong superconductivity, we have $R_{\mathrm{tot}}^{\perp}(y, r)=4 \pi^{2} r /[45 \zeta(3) y]$.

We have calculated $R_{\text {tot }}^{\perp}(y, r)$ on a dense grid of $y$ and $r$ and fitted the results by the formula

$$
\begin{aligned}
R_{\mathrm{tot}}^{\perp}(y, r) & =p_{1} \exp \left(-0.14 y^{2}\right)+\frac{1-p_{1}}{\sqrt{1+p_{3} y^{2}}}, \\
p_{1} & =0.48-0.17 r \\
p_{3} & =\left[\left(1-p_{1}\right) \frac{45 \zeta(3)}{4 \pi^{2} r}\right]^{2},
\end{aligned}
$$

which reproduces also the asymptote of $R_{\mathrm{tot}}^{\perp}(y, r)$ at $y \rightarrow \infty$ and the obvious condition $R_{\text {tot }}^{\perp}(0, r)=1$. The maximum fit error $\approx 5 \%$ takes place at $y=1.2$ and $r=1$.

For the longitudinal components of the collision frequencies $\nu_{e p}^{\|}$and $\nu_{\mu p}^{\|}$, the angular integration in (25) remains unchanged. Then

$$
\nu_{c p}^{\| S}=\nu_{c p}^{\|} R_{p}^{\|}(y)
$$

where $\nu_{c p}^{\|}$is the nonsuperconducting value, while

$$
\begin{aligned}
R_{p}^{\|}(y)= & \frac{15}{4 \pi^{4}} \int_{0}^{\infty} \int_{0}^{\infty} \frac{\mathrm{d} x_{2} \mathrm{~d} x_{2}^{\prime}}{1+\exp \left(z_{2}\right)} \\
& \times\left\{\frac{\left(z_{2}^{\prime}-z_{2}\right)^{3}\left(1-4 \mathrm{u}_{2} \mathrm{u}_{2}^{\prime} \mathrm{v}_{2} \mathrm{v}_{2}^{\prime}\right)}{\left[1+\exp \left(-z_{2}^{\prime}\right)\right]\left[\exp \left(z_{2}^{\prime}-z_{2}\right)-1\right]}\right. \\
& \left.-\frac{\left(z_{2}^{\prime}+z_{2}\right)^{3}\left(1+4 \mathrm{u}_{2} \mathrm{u}_{2}^{\prime} \mathrm{v}_{2} \mathrm{v}_{2}^{\prime}\right)}{\left[1+\exp \left(z_{2}^{\prime}\right)\right]\left[\exp \left(-z_{2}^{\prime}-z_{2}\right)-1\right]}\right\}
\end{aligned}
$$

is the superfluid reduction factor.

In the limit of $y \gg 1$ we obtain $R_{p}^{\|}(y) \rightarrow A_{\|} \exp (-y)$, where

$$
\begin{aligned}
A_{\|}= & \frac{15}{2 \pi^{4}} \int_{0}^{\infty} \mathrm{d} \eta_{1} \int_{0}^{\infty} \mathrm{d} \eta_{2} \\
& \times \frac{\left(\eta_{1}^{2}-\eta_{2}^{2}\right)^{3}\left(\eta_{1}^{2}+\eta_{2}^{2}\right)}{\exp \left(\eta_{1}^{2}\right)-\exp \left(\eta_{2}^{2}\right)} \approx 1.00 .
\end{aligned}
$$

Finally, in analogy with Eq. (88), we have numerically calculated $R_{p}^{\|}(y)$ and fitted it by

$$
\begin{aligned}
R_{p}^{\|}(y) & =\left\{1+\left(26.33 y^{2}+0.376 y^{4}\right)\right. \\
& \times \exp \left(-\sqrt{(3.675)^{2}+y^{2}}\right) \\
& \left.+0.742\left[\exp \left((1.673)^{2}-\sqrt{(1.673)^{2}+y^{2}}\right)-1\right]\right\} \\
& \times \exp \left((1.361)^{2}-\sqrt{(1.361)^{2}+y^{2}}\right)
\end{aligned}
$$

with the error $\lesssim 1 \%$.

The reduction factor $R_{p}^{\|}(y)$ was introduced by Gnedin and Yakovlev [17] [see their Eq. (43)]. In contrast to our Eq. (90), their reduction factor had two drawbacks. First, it neglected coherence factors - the terms containing $\mathrm{u}_{2}$ and $\mathrm{v}_{2}$ in (90). Second, the authors of Ref. [17] attributed the same reduction $R_{p}^{\|}(y)$ to collisions via the exchange of transverse and longitudinal plasmons [whereas in fact 
the reduction factors $R_{p}^{\perp}(y, r)$ and $R_{p}^{\|}(y)$ are different]. However, because superfluid reduction of $c p$ collisions for $y \gg 1$ is exponentially strong, these drawbacks do not change noticeably numerical values of the thermal conductivity (while neglecting the exchange of transverse plasmons makes results really inaccurate).

Proton superconductivity significantly modifies the thermal conductivity of electrons and muons. In particular, it violates the temperature independence of the conductivity (40) for nonsuperconducting matter. The asymptote (87) indicates that in a strongly superconducting matter $\left(T \ll T_{c p}, y \gg 1\right)$ the collision frequency $\nu_{c}^{\perp S}$ becomes much smaller than in the nonsuperconducting case. Strong superconductivity enforces the temperature dependence $\nu_{c}^{\perp S} \propto T^{2}$ that is formally the same as for the longitudinal collision frequency $\nu_{c}^{\|}$in normal Fermi liquid. Nevertheless, this thermal conductivity remains smaller than the conductivity in the normal matter if it included artificially the exchange of longitudinal plasmons alone.

As in normal matter, the major contribution to the electron or muon thermal conductivity in the presence of proton superconductivity comes from the exchange of transverse plasmons. Using the asymptotes for the reduction factors in the limit of strong superconductivity $(y \gg 1)$ we obtain (in standard physical units)

$$
\kappa_{c}^{S} \approx \kappa_{c}^{S \perp} \approx \frac{5}{24} \frac{k_{B} c p_{F c}^{2}}{\alpha \hbar^{2}} \frac{\Delta}{k_{B} T} \frac{p_{F p}^{2}}{p_{F e}^{2}+p_{F \mu}^{2}} .
$$

Therefore, strong proton superconductivity formally restores the temperature dependence of the thermal conductivity $\kappa_{e \mu} \propto 1 / T$ typical for a normal Fermi liquid (by enforcing nondissipative plasma screening). Accordingly, we expect that in this case our simplest variational thermal conductivity is different from the exact conductivity by the same factor $C \approx 1.2$ that is characteristic for Coulomb scattering via the exchange of longitudinal plasmons [17]. These arguments support our expectations (Sec. IIC) that the overall uncertainties of our calculations of $\kappa_{e \mu}$ are $\sim 20 \%$. Notice that $\kappa_{e \mu}^{S}$ is proportional to the superfluid gap $\Delta$.

\section{RESULTS AND DISCUSSION}

\section{A. Equations of state}

Let us illustrate our results by calculating the thermal conductivity in a neutron star core composed of neutrons, protons, electrons and muons. We employ three representative equations of state (EOSs) which we denote as APR, PAL II, and PAL IV. The fractions of electrons $x_{e}=n_{e} / n_{b}$ and muons $x_{\mu}=n_{\mu} / n_{b}$ versus density of matter $\rho_{14}=\rho /\left(10^{14} \mathrm{~g} \mathrm{~cm}^{-3}\right)$ for these EOSs are plotted in Fig. $2\left(n_{b}=n_{n}+n_{p}\right.$ being the number density of baryons). Let us recall that the lowest density in a neutron star core is $\rho_{\text {min }} \approx 1.5 \times 10^{14} \mathrm{~g} \mathrm{~cm}^{-3}$.

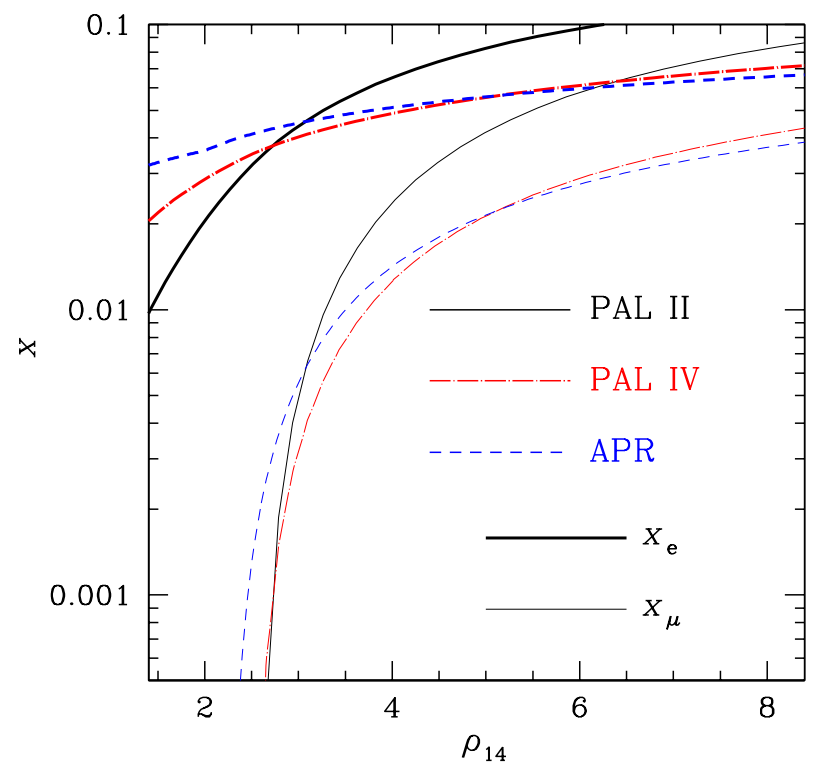

Figure 2: (Color online) Fractions of electrons (thick lines) and muons (thin lines) versus density in a neutron star core for three selected EOSs - APR (dashed lines), PAL II (solid lines), and PAL IV (dash-dot lines).

The APR EOS was constructed by Akmal, Pandharipande and Ravenhall [33] (their model Argonne V18 + $\left.\delta v+\mathrm{UIX}^{*}\right)$. It is sufficiently stiff and gives the maximum gravitational mass of stable neutron stars $M_{\max }=$ $2.2 M_{\odot}$. The central density of the maximum-mass star is $\rho_{\max }=2.8 \times 10^{15} \mathrm{~g} \mathrm{~cm}^{-3}$. No hyperons appear for this $\mathrm{EOS}$ at $\rho \leq \rho_{\max }$. The threshold density for the appearance of muons is $\rho_{\mu} \approx 2.28 \times 10^{14} \mathrm{~g} \mathrm{~cm}^{-3}$.

The PAL II is a convenient phenomenological semianalytical model EOS proposed by Prakash, Ainsworth and Lattimer [34]. The authors suggested several EOSs of such a type which differ by a value of the compression modulus $K_{0}$ of saturated symmetric nuclear matter and by the symmetry energy $S$ of dense matter as a function of baryon number density $n_{b}$ [described by a function $F(u)$, where $u=n_{b} / n_{0}$ and $n_{0} \approx 0.16 \mathrm{fm}^{-3}$ is the baryon number density in saturated symmetric nuclear matter]. The compression modulus determines the stiffness of an EOS, while the symmetry energy regulates the fraction of protons (and hence of electrons and muons). The PAL II EOS corresponds to $F(u)=2 u^{2} /(u+1)$ which gives a rather high fraction of protons at $u>1$ and the muon threshold density $\rho_{\mu}=2.647 \times 10^{14} \mathrm{~g} \mathrm{~cm}^{-3}$ (Fig. 2). Number densities of various particles, and hence $\kappa_{e \mu}$, are insensitive to the value of $K_{0}$ for PAL EOSs 34]. Notice that by taking $K_{0}=120,180$, and $240 \mathrm{MeV}$ (as suggested in Ref. [34]), we would obtain three modifications of the PAL II EOS with different compressibility (from soft to stiff) which would give very different neutron star models (different mass-radius relations and maximum masses).

The PAL IV EOS belongs to the same PAL family of EOSs 34] but corresponds to the function $F(u)=u^{0.7}$ 


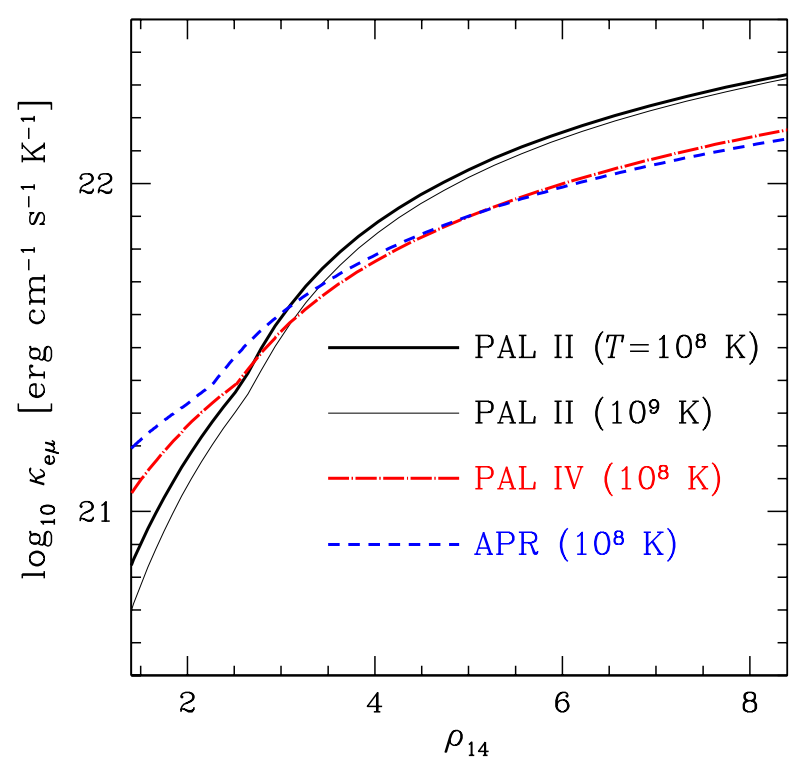

Figure 3: (Color online) Electron-muon thermal conductivity $\kappa_{e \mu}$ versus density in a nonsuperconducting neutron star core. Thick lines are for the APR (dashed line), PAL II (solid line) and PAL IV (dot-dashed line) EOSs at $T=10^{8} \mathrm{~K}$. Thin solid line is for the PAL II EOS at $T=10^{9} \mathrm{~K}$.

suggested by Page and Applegate [35]. It gives noticeably lower fractions of protons, muons and electrons at $u>1$ than those provided by the PAL II EOS (Fig. 2). The muon creation threshold is $\rho_{\mu}=2.51 \times 10^{14} \mathrm{~g} \mathrm{~cm}^{-3}$. Taking again different $K_{0}$ we get different modifications of the PAL IV EOS, from soft to stiff, giving different neutron star models.

Therefore, the selected EOSs cover a large range of physical models of npe $\mu$ matter in neutron star cores.

\section{B. Thermal conductivity in nonsuperconducting cores}

Figure 3 shows the density dependence of the full electron-muon thermal conductivity $\kappa_{e \mu}$ for the selected EOSs. The thick lines give $\kappa_{e \mu}$ at $T=10^{8} \mathrm{~K}$, a typical internal temperature of a middle-aged neutron star $\left(t \sim 10^{4}-10^{5} \mathrm{yr}\right)$ with no enhanced neutrino emission generated in its core (see, e.g., Ref. [5]). The conductivity $\kappa_{e \mu}$ increases with growing $\rho$ because of the increasing amount of electrons and muons in dense matter [see Eq. (40)]. The conductivity shows a noticeable kink at $\rho=\rho_{\mu}$ associated with the appearance of muons. The conductivities for the APR and PAL IV EOSs are close to each other because these two EOSs give very similar amounts of electrons and muons (Fig. (2). At $\rho \gtrsim 4 \times 10^{14} \mathrm{~g} \mathrm{~cm}^{-3}$, the conductivity for the PAL II EOS is noticeably higher owing to larger fractions of electrons and muons for the PAL II EOS at high densities.

The thin solid line in Fig. 3 displays $\kappa_{e \mu}$ for the PAL

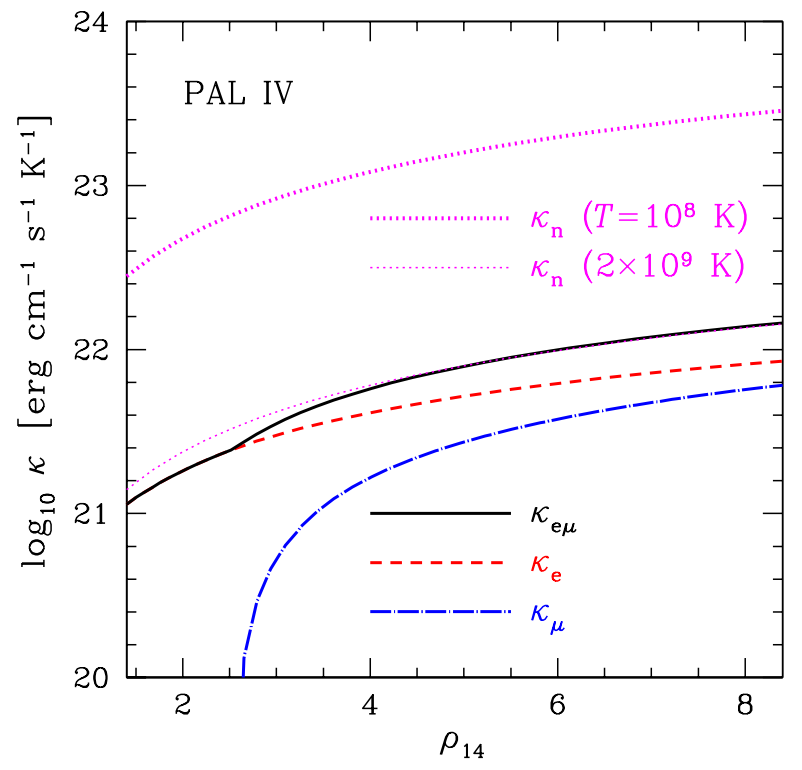

Figure 4: (Color online) Electron (dashed line), muon (dotdashed line), electron-muon (solid line) thermal conductivities (calculated for $T=10^{8} \mathrm{~K}$ but almost temperature independent) and neutron conductivity (for $T=10^{8}$ and $2 \times 10^{9} \mathrm{~K}$, thick and thin dotted lines, respectively) versus density in a normal neutron star core for the PAL IV EOS.

II EOS at a higher temperature $T=10^{9} \mathrm{~K}$ typical of a young neutron star (a few months after its birth in the absence of enhanced neutrino emission; e.g., Ref. [5]). It is very close to the conductivity at $T=10^{8} \mathrm{~K}$ confirming our prediction (40) that $\kappa_{e \mu}$ is almost independent of temperature in nonsuperconducting matter because of the importance of the Landau damping. The Landau damping owing to the exchange of transverse plasmons is the dominant mechanism of electron and muon scattering; it fully regulates $\kappa_{e \mu}$ in nonsuperconducting neutron star cores (Sec. IIB).

Figure 4 compares the thermal conductivity provided by different particles in a nonsuperfluid neutron star for the PAL IV EOS. We show the density dependence of the electron conductivity $\kappa_{e}$, the muon conductivity $\kappa_{\mu}$, and the electron-muon conductivity $\kappa_{e \mu}$ calculated at $T=10^{8} \mathrm{~K}$ with the notice that these conductivities are almost temperature independent. We also plot the neutron conductivity $\kappa_{n}$ calculated using the results of Baiko, Haensel and Yakovlev [15] for $T=10^{8} \mathrm{~K}$ (the thick dotted line) and $2 \times 10^{9} \mathrm{~K}$ (the thin dotted line). It is determined by collisions of neutrons with neutrons and protons via nuclear interactions and demonstrates a traditional Fermi liquid behavior $\left(\kappa_{n} \propto T^{-1}\right)$. The effective masses of neutrons and protons are set equal to 0.7 of their bare masses, and the in-medium effects on the squared matrix elements of nucleon-nucleon scattering are neglected. It is seen that the neutron contribution dominates throughout the neutron star core at $T=10^{8} \mathrm{~K}$ (contrary to the previous results [13, 17]). However, we 


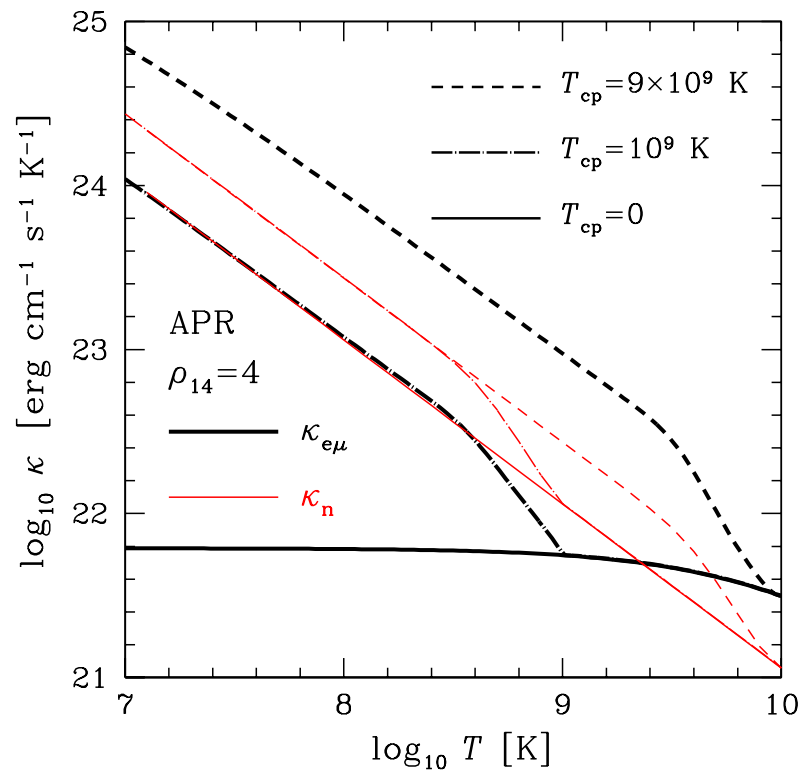

Figure 5: (Color online) Temperature dependence of the electron-muon thermal conductivity $\kappa_{e \mu}$ (thick lines) and the neutron conductivity $\kappa_{n}$ (thin lines) in a neutron star core with the APR EOS at $\rho=4 \times 10^{14} \mathrm{~g} \mathrm{~cm}^{-3}$. Solid lines refer to nonsuperconducting matter, while dot-dashed and dashed lines are for matter with proton superconductivity (with $T_{c p}=10^{9}$ and $9 \times 10^{9} \mathrm{~K}$, respectively).

have $\kappa_{e \mu} \gtrsim \kappa_{n}$ in the core of a hot neutron star with $T \gtrsim 2 \times 10^{9} \mathrm{~K}$.

\section{Thermal conductivity in superconducting matter}

Let us illustrate the effects of proton superconductivity on the electron-muon thermal conductivity $\kappa_{e \mu}$. Figure 5 shows $\kappa_{e \mu}$ and $\kappa_{n}$ versus temperature for the APR EOS at $\rho=4 \times 10^{14} \mathrm{~g} \mathrm{~cm}^{-3}$ assuming either $T_{c p}=0$ (normal matter; solid lines), or $T_{c p}=10^{9} \mathrm{~K}$ (dot-dashed lines), or $T_{c p}=9 \times 10^{9} \mathrm{~K}$ (dashed lines). The neutron conductivity is calculated using the results of Ref. [15] under the assumption that neutrons are normal.

In normal matter $\kappa_{n}$ dominates over $\kappa_{e \mu}$ at $T \lesssim$ $2 \times 10^{9} \mathrm{~K}$ in agreement with the results of Sec. IVB. We see that $\kappa_{e \mu}$ is almost temperature independent because of the dominant contribution of the Landau damping [Eq. (40)]. Proton superconductivity reduces the electron and muon collision frequencies and increases $\kappa_{e u}$ as discussed in Sec. III, it also slightly increases $\kappa_{n}$ [15]. Therefore, when the temperature drops below $T_{c p}$ and proton superconductivity sets in, $\kappa_{e \mu}$ starts to grow up much quicker than $\kappa_{n}$ and becomes comparable to or larger than $\kappa_{n}$. At $T \lesssim T_{c p} / 3$ this increase of $\kappa_{e \mu}$ is well described by the asymptotic expression (93), $\kappa_{e \mu} \propto T^{-1}$. Proton superconductivity formally restores the Fermi liquid behavior of $\kappa_{e \mu}$ (Sec. III). However, this $\kappa_{e \mu}$ is smaller than the electron-muon conductivity which is calculated in Refs. 13, 17]. Nevertheless, because $\kappa_{e \mu}$ in superconducting matter is directly proportional to the proton gap $\Delta \propto T_{c p}$, the enhancement of $\kappa_{e \mu}$ by proton superconductivity makes $\kappa_{e \mu}$ quite large. According to Fig. 5. for $T_{c p}=10^{9} \mathrm{~K}$ and $T \lesssim 3 \times 10^{8} \mathrm{~K}$ we have $\kappa_{e \mu}$ much larger than in normal matter although a factor of $\sim 3$ lower than $\kappa_{n}$. For $T_{c p}=3 \times 10^{9} \mathrm{~K}$ (not shown in the figure) we would have $\kappa_{e \mu} \sim \kappa_{n}$ at any $T$ displayed. For a stronger superconductivity with $T_{c p}=9 \times 10^{9} \mathrm{~K}$, as seen from Fig. 5. $\kappa_{e \mu}$ dominates over $\kappa_{n}$ at any $T$. Therefore, we obtain $\kappa_{e \mu} \gtrsim \kappa_{n}$ for $T \gtrsim 2 \times 10^{9} \mathrm{~K}$ in normal matter and for any $T$ in superconducting matter with $T_{c p} \gtrsim 3 \times 10^{9} \mathrm{~K}$.

\section{CONCLUSIONS}

We have calculated the thermal conductivity of electrons and muons $\kappa_{e \mu}$ in the cores of neutron stars composed of neutrons, protons, electrons, and muons taking into account possible superconductivity of protons. This thermal conductivity is determined by electromagnetic interactions of electrons and muons with all charged particles. Our results are presented in the form of simple analytic expressions and fitting formulae valid for any equation of state of dense matter in a neutron star core and for any ratio $T / T_{c p}$ between the temperature $T$ and proton critical temperature $T_{c p}$ in superconducting matter $\left(T<T_{c p}\right)$. A generalization of our results to hyperonic matter (including the case of superfluid hyperons) is straightforward (analogous to that in Ref. [17]).

Our main conclusions are as follows.

1. The main contribution to $\kappa_{e \mu}$ comes from electromagnetic interactions of charged particles via the exchange of transverse plasmons. This contribution has been neglected in all previous calculations of $\kappa_{e \mu}$ in neutron star cores (although it has been included in the calculation of the thermal conductivity of quarks in quark matter [18] and the thermal conductivity of electrons in neutron star crusts [19]).

2. For normal (nonsuperconducting) protons, $\kappa_{e \mu}$ is determined by electromagnetic interactions of electrons and muons with all charged particles $(e, \mu$, $p$ ) via the Landau damping of transverse plasmons [Eq. (40)]. This thermal conductivity is temperature independent (contrary to the traditional Fermi liquid behavior $\left.\kappa \propto T^{-1}\right)$.

3. The conductivity $\kappa_{e \mu}$ is mainly determined by the symmetry energy of dense matter (rather than by the stiffness of the equation of state) and increases with the growth of the symmetry energy.

4. At temperatures $T \sim 10^{8} \mathrm{~K}$, the conductivity $\kappa_{e \mu}$ in a normal neutron star core is smaller than the conductivity of neutrons $\kappa_{n}$, but at $T \gtrsim 2 \times 10^{9} \mathrm{~K}$ we obtain $\kappa_{e \mu} \gtrsim \kappa_{n}$. 
5. After the onset of proton superconductivity (at $\left.T<T_{c p}\right) \kappa_{e \mu}$ remains to be determined by electromagnetic interactions of charged particles via the exchange of transverse plasmons but the character of these interactions is different. First, strong proton superconductivity greatly suppresses the collisions of electrons and muons with protons. Second, this superconductivity modifies the exchange of transverse plasmons (from the dissipative Landau damping to the nondissipative regime). As a result, strong superconductivity $\left(T \lesssim T_{c p} / 3\right)$ increases $\kappa_{e \mu}$, restores its Fermi liquid behavior, and enforces $\kappa_{e \mu}$ to scale as $\kappa_{e \mu} \propto T_{c p} / T$. For $T_{c p} \gtrsim 3 \times 10^{9} \mathrm{~K}$, we obtain $\kappa_{e \mu} \gtrsim \kappa_{n}$ at any $T$.

Our results are based on previous calculations of Gnedin and Yakovlev [17]. We have extended them by properly including the charged particle interactions via the exchange of transverse plasmons and by performing exact calculation of the rate of electron/muon scattering by proton quasiparticles in the presence of proton superconductivity.

We expect that our results give reliable values of $\kappa_{e \mu}$ in neutron star cores. In the absence of proton superconductivity, calculation of $\kappa_{e \mu}$ constitutes a well defined problem, which we solve explicitly (under the only one and well justified assumption of weak plasma screening). Because the solution involves only electromagnetic interactions, it is universal. It does not depend on a strong interaction model of dense matter and, hence, on a specific equation of state in a neutron star core. In the presence of proton superconductivity, we use the polarization functions of a proton gas derived in the BCS framework. We argue (Sec. III A) that it can be a good approximation. Even if future studies of realistic nucleon Fermi liquid give more elaborated polarization functions, our solution will serve as a useful basic approximation.

In any case our results do not solve the thermal conduction problem in neutron star cores. The main problem is posed by the conductivity of neutrons and protons (and other baryons if available) mediated by strong (nuclear) interactions, especially in the presence of baryon superfluidity. The conductivities of neutrons and protons, $\kappa_{n}$ and $\kappa_{p}$, in normal matter have been estimated by Flowers and Itoh [13, 14] (with the natural result that $\kappa_{p}$ is much smaller than $\kappa_{n}$ because of smaller amount of protons in neutron star cores). More accurate estimations of $\kappa_{n}$ were done later by Baiko et al. [15] who estimated also the diffusive thermal conductivity $\kappa_{n}$ in the presence of neutron and proton superfluidity. However, the appearance of neutron superfluidity can trigger a specific and efficient nondiffusive heat conduction via convective counterflow. This effect is well known from laboratory experiments with superfluid ${ }^{4} \mathrm{He}$ (e.g., Ref. [36]); it is so efficient that immediately dissolves any temperature gradients in superfluid ${ }^{4} \mathrm{He}$. Analogous effects in superfluid neutron star cores have been mentioned in the astrophysical literature (e.g., Ref. [13]) but have not been explored. Their careful examination would be desirable.

It would also be important to study the effects of strong magnetic fields which can greatly modify thermal conductivity in neutron star cores. Strong Larmor rotation of charged particles (electrons, muons, protons) about magnetic field lines can greatly suppress thermal conduction across the magnetic field. Similar effects in electric conductivity have been studied in a number of works (see, e.g., 37] and references therein) but they are almost unexplored for the thermal conductivity. The magnetic field modifies plasmon modes in dense matter and affects plasma polarization properties, particularly, the Landau damping. The magnetic field effects can be especially complicated in the presence of baryon superfluidity.

All in all, further serious efforts are required to solve the heat conduction problem in neutron star cores. First of all, its solution is needed to model cooling of neutron stars 5, 6] (especially in the first 100 years of their life [10, 11]), thermal states of neutron stars in soft X-ray transients [6, 7, 8, 9] and thermal relaxation of pulsars after glitches [12]. We hope that our results give a reliable contribution to the thermal conduction problem, and we expect to study other effects of the Landau damping on kinetic properties of neutron star cores (particularly, on the shear viscosity) in subsequent publications.

\section{Acknowledgments}

We are extremely grateful to M. E. Gusakov for many useful discussions and critical remarks. This work was partly supported by the Dynasty Foundation, by the Russian Foundation for Basic Research (grants 05-02-16245, 05-02-22003), and by the Federal Agency for Science and Innovations (grant NSh 9879.2006.2).
[1] S. L. Shapiro and S. A. Teukolsky, Black Holes, White Dwarfs, and Neutron Stars (Wiley-Interscience, New York, 1983).

[2] J. M. Lattimer and M. Prakash, Astrophys. J. 550, 426 (2001).

[3] P. Haensel, in Final Stages of Stellar Evolution, edited by C. Motch and J.-M. Hameury (EAS Publ. Ser., EDP Sci., 2003) p. 249.
[4] J. M. Lattimer and M. Prakash, Science 304, 536 (2004).

[5] D. G. Yakovlev and C. J. Pethick, Annu. Rev. Astron. Astrophys. 42, 169 (2004).

[6] D. Page, U. Geppert, and F. Weber, Nucl. Phys. A 777, 497 (2006).

[7] Brown E. F., Bildsten L., and Rutledge R. E., Astrophys. J. Lett. 504, L95 (1998).

[8] Haensel P. and Zdunik J. L., Astron. Astrophys. 227, 
431 (1990).

[9] Haensel P. and Zdunik J. L., Astron. Astrophys. 404, L33 (2003).

[10] J. M. Lattimer, K. A. Van Riper, M. Prakash, and M. Prakash, Astrophys. J. 425, 802 (1994).

[11] O. Y. Gnedin, D. G. Yakovlev, and A. Y. Potekhin, Mon. Not. R. Astron. Soc. 324, 725 (2001).

[12] M. B. Larson and B. Link, Mon. Not. R. Astron. Soc. 333, 613 (2002).

[13] E. Flowers and N. Itoh, Astrophys. J. 230, 847 (1979).

[14] E. Flowers and N. Itoh, Astrophys. J. 250, 750 (1981).

[15] D. A. Baiko, P. Haensel, and D. G. Yakovlev, Astron. Astrophys. 374, 151 (2001).

[16] E. Flowers and N. Itoh, Astrophys. J. 206, 218 (1976).

[17] O. Y. Gnedin and D. G. Yakovlev, Nucl. Phys. A 582, 697 (1995).

[18] H. Heiselberg and C. J. Pethick, Phys. Rev. D 48, 2916 (1993).

[19] P. S. Shternin and D. G. Yakovlev, Phys. Rev. D 74, 043004 (2006).

[20] P. Jaikumar, C. Gale, and D. Page, Phys. Rev. D 72, 123004 (2005).

[21] V. B. Berestetskiŭ, E. M. Lifshitz, and L. P. Pitaevskii, Quantum Electrodynamics (ButterworthHeinemann, Oxford, 1982).

[22] A. F. Alexandrov, L. S. Bogdankevich, and A. A. Rukhadze, Principles of Plasma Electrodynamics (Springer-Verlag, Berlin, Heidelberg, New York, Tokyo, 1984), Springer Series in Electrophysics, Vol. 9.

[23] E. M. Lifshitz and L. P. Pitaevskiı̌, Statistical Physics, Part 2 (Pergamon, Oxford, 1980).
[24] J. Sykes and G. A. Brooker, Ann. Phys. (NY) 56, 1 (1970).

[25] R. H. Anderson, C. J. Pethick and K. F. Quader, Phys. Rev. B 35, 1620 (1987).

[26] G. Baym and C. J. Pethick, Landau Fermi-Liquid Theory. Concepts and Applications (Wiley, New-York, 1991).

[27] U. Lombardo and H.-J. Schulze, in Physics of Neutron Star Interiors, edited by D. Blaschke, N. Glendenning, and A. Sedrakian (Springer, Berlin, 2001), p. 30.

[28] K. P. Levenfish and D. G. Yakovlev, Astron. Rep. 38, 247 (1994).

[29] P. I. Arseev, S. O. Loiko, and N. K. Fedorov, Usp. Fiz. Nauk 49, 1 (2006) [Phys. — Usp. 49, 1 (2006)].

[30] J. Kundu and S. Reddy, Phys. Rev. C 70, 055803 (2004).

[31] D. C. Mattis and J. Bardeen, Phys. Rev. 111, 412 (1958).

[32] A. A. Abrikosov, L. P. Gorkov, and I. E. Dzyaloshinski, Methods of Quantum Field Theory in Statistical Physics (Courier Dover Publications, 1975).

[33] A. Akmal, V. R. Pandharipande, and D. G. Ravenhall, Phys. Rev. C 58, 1804 (1998).

[34] M. Prakash, T. L. Ainsworth, and J. M. Lattimer, Phys. Rev. Lett. 61, 2518 (1988).

[35] D. Page and J. H. Applegate, Astrophys. J. Lett. 394, L17 (1992).

[36] D. R. Tilley and J. Tilley, Superfluidity and Superconductivity (IOP Publishing, Bristol, 1990).

[37] D. G. Yakovlev, in Strongly Coupled Plasma Physics, edited by H. M. Van Horn and S. Ichimaru (University of Rochester, Rochester, 1993), p. 157. 